\title{
Discrimination of Four Marine Biofilm-Forming Bacteria by LC-MS Metabolomics and Influence of Culture Parameters
}

\author{
Laurie Favre, ${ }^{\dagger}$ Annick Ortalo-Magné, Stéphane Greff, ${ }^{\dagger}$ Thierry Pérez, ${ }^{\ddagger}$ Olivier P. Thomas, \\ Jean-Charles Martin, " and Gérald Culioli* \\ ${ }^{\dagger}$ Université de Toulon, MAPIEM, EA 4323, La Garde Cedex 83130, France \\ ${ }^{\ddagger}$ CNRS, Aix Marseille Univ, IRD, Avignon Univ. Institut Méditerranéen de Biodiversité et d’Ecologie marine et continentale, Station \\ marine d'Endoume, Marseille 13007, France \\ ${ }^{\S}$ National University of Ireland Galway, School of Chemistry, Marine Biodiscovery, Galway, Ireland \\ "Aix-Marseille Univ, INSERM, INRA, NORT, Marseille 13005, France
}

\begin{abstract}
Most marine bacteria can form biofilms, and they are the main components of biofilms observed on marine surfaces. Biofilms constitute a widespread life strategy, as growing in such structures offers many important biological benefits. The molecular compounds expressed in biofilms and, more generally, the metabolomes of marine bacteria remain poorly studied. In this context, a nontargeted LC-MS metabolomics approach of marine biofilm-forming bacterial strains was developed. Four marine bacteria, Persicivirga (Nonlabens) mediterranea TC4 and TC7, Pseudoalteromonas lipolytica TC8, and Shewanella sp. TC11, were used as model organisms. The main objective was to search for some strainspecific bacterial metabolites and to determine how culture parameters (culture medium, growth phase, and mode of culture) may affect the cellular metabolism of each strain and thus the global interstrain metabolic discrimination. LC-MS profiling and statistical partial least-squares discriminant analyses showed that the four strains could be differentiated at the species level whatever the medium, the growth phase, or the mode of culture (planktonic vs biofilm). A MS/MS molecular network was subsequently built and allowed the identification of putative bacterial biomarkers. TC 8 was discriminated by a series of ornithine lipids, while the $P$. mediterranea strains produced hydroxylated ornithine and glycine lipids. Among the $P$. mediterranea strains, TC7 extracts were distinguished by the occurrence of diamine derivatives, such as putrescine amides.
\end{abstract}

KEYWORDS: marine bacteria, biofilms, metabolomics, liquid chromatography-mass spectrometry, MS/MS networking, ornithine lipids, polyamines

\section{INTRODUCTION}

All biotic or abiotic surfaces immersed in the marine environment are subjected to colonization pressure by a great diversity of micro- and macroorganisms (e.g., bacteria, diatoms, micro- and macroalgae, invertebrate larvae). This so-called "marine biofouling" generates serious economic issues for endusers of the marine environment. Biofouling drastically alters boat hulls, pipelines, aquaculture, and port structures, ${ }^{1}$ thus affecting fisheries and the maritime industry by reducing vessel efficiency and increasing maintenance costs. ${ }^{2}$ Among fouling organisms, bacteria are well known for their significant pioneer role in the process of colonization. ${ }^{3}$ They are commonly considered as the first colonizers of immersed surfaces. They organize themselves in communities called biofilms, forming complex structures of cells embedded in an exopolymeric matrix. ${ }^{4}$ Thousands of bacterial strains are present in marine biofilms, and bacterial cell concentration is higher than in planktonic samples isolated from the same environment. Such an organization confers a special functioning to the prokaryotic community: ${ }^{5}$ (i) it provides a better resistance to exogenous stresses, (ii) it allows nutrients to accumulate at the surface, and (iii) it can constitute a protective system to predation. ${ }^{6}$ Moreover, the composition of the community and its biochemical production have been shown to impact the settlement of other organisms and thus the maturation of the biofouling. 7,8

From a chemical point of view, marine bacteria are known to produce a wide array of specialized metabolites exhibiting various biological activities. ${ }^{9}$ Among them, a vast number of compounds serve as protectors in highly competitive environments, and others have specific roles in physiology, communication, or constitute adaptive responses to environmental changes. ${ }^{10}$ Therefore, obtaining broad information on 
the metabolic status of bacterial strains isolated from marine biofilms and correlating it with external parameters is of high interest. Such knowledge constitutes a prerequisite for further studies on the overall understanding of these complex ecological systems.

With the recent developments of metabolomics, it is now possible to obtain a snapshot view, as complete and accurate as possible, of a large set of metabolites (i.e., small organic molecules with $M_{\mathrm{w}}<1500 \mathrm{Da}$ ) in a biological sample reflecting the metabolic state of the cells as a result of the specificity of their genetic background and an environmental context. Nuclear magnetic resonance spectroscopy or hyphenated techniques such as liquid chromatography (LC) or gas chromatography (GC) coupled to mass spectrometry are commonly used as analytical tools for metabolomics studies. Liquid chromatography-mass spectrometry (LC-MS) has the advantage to analyze a large pool of metabolites with high sensitivity and resolution, even without derivatization. ${ }^{11}$ In comparative experiments, metabolomics applied to bacteria allows the identification of biomarkers able to differentiate strains. To date, a limited number of metabolomics studies have focused on marine bacteria, and only few of them are related to the effects of physiological and culture parameters on bacterial metabolism. ${ }^{12-15}$

With the main objectives to search for some strain-specific bacterial metabolites and to assess the influence of culture parameters on the strain metabolism, this study intended: (i) to evaluate the LC-MS-based discrimination between the metabolome of four marine biofilm-forming bacterial strains depending on different extraction solvents and culture conditions and (ii) to putatively annotate the main discriminating compounds (Figure 1).

The four marine strains studied herein are all Gram-negative bacteria isolated from natural biofilms: Persicivirga (Nonlabens) mediterranea TC4 and TC7 belong to the phylum Bacteroidetes, while Pseudoalteromonas lipolytica TC8 and Shewanella sp. TC11 are $\gamma$-proteobacteria. They were selected on the basis of their biofilm-forming capability when cultivated in vitro and their ease for growing. ${ }^{16}$ The two first strains (TC4 and TC7) were specifically chosen to evaluate the discriminative potential of our metabolomics approach as they belong to the same species. Because of the use of high-salt culture media when working on marine bacteria and to obtain an efficient extraction of intracellular metabolites, liquid-liquid extraction with medium polarity agents was specifically selected. For the analytical conditions, C18 reversed-phase HPLC columns are widely used for LC-MS profiling. ${ }^{17}$ Such separation process provides satisfactory retention of medium to low polar analytes but does not allow a proper retention of more polar compounds. In the present study, analyses were performed on a phenyl-hexyl stationary phase to detect a large array of bacterial metabolites. The recently developed core-shell stationary phase was applied here for improved efficiency. ${ }^{18}$ For the MS detection, even if high-resolution mass spectrometry (HRMS) is mainly used in metabolomics, a low-resolution mass spectrometer (LRMS) was first chosen to assess the potential of the metabolomic approach to discriminate between the bacteria. A cross-platform comparison including HRMS was subsequently undertaken to assess the robustness of the method. Finally, HRMS and MS/MS data were used for the metabolite annotation. The resulting data were analyzed by multivariate statistical methods, including principal component analysis (PCA) and supervised partial
4 marine biofilm-forming bacteria

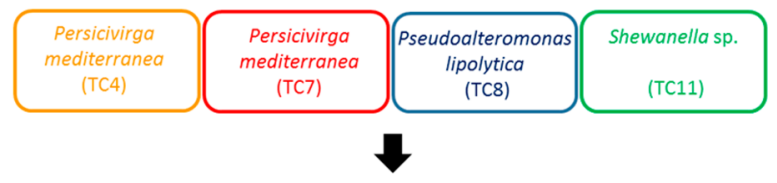

\section{Impact on their metabolome of extraction \& culture conditions}

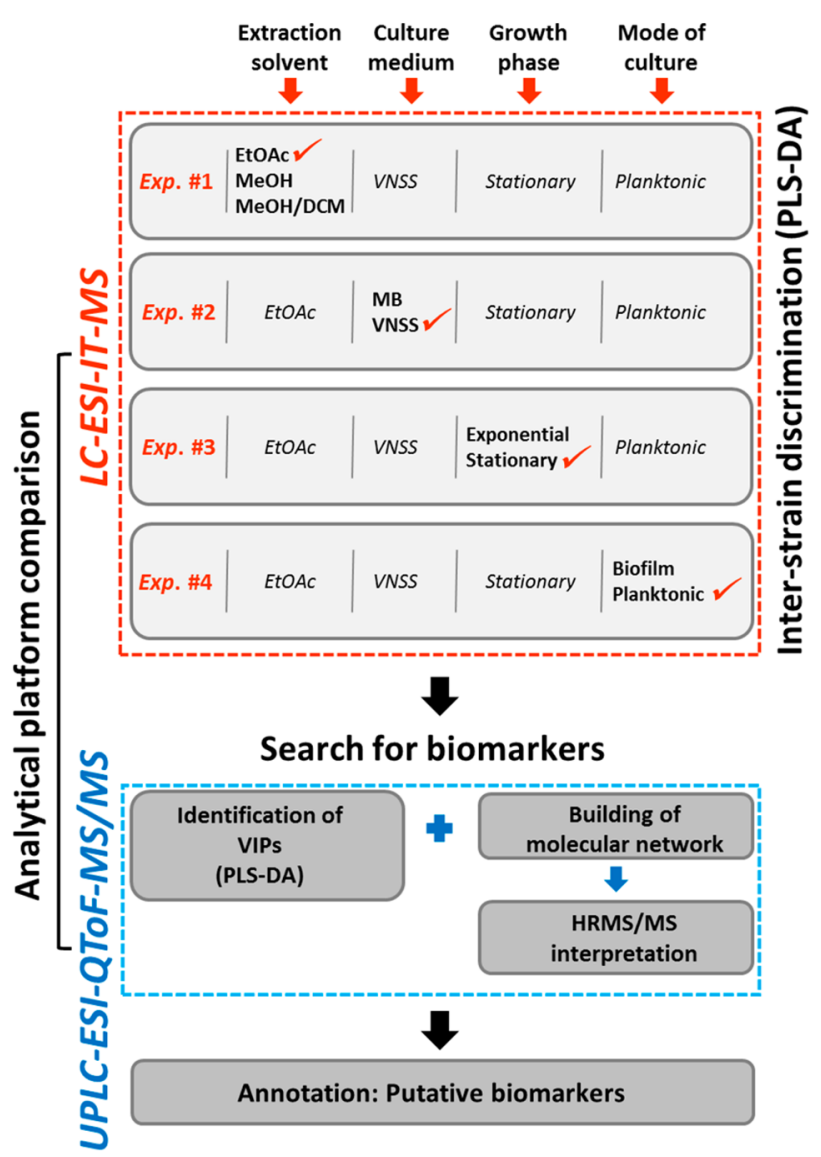

Figure 1. Overview of the experimental workflow used for the discrimination of the four marine bacterial strains and for the putative identification of relevant biomarkers.

least-squares discriminate analysis (PLS-DA). Unsupervised PCA models were first used to evaluate the divide between bacterial strains, while supervised PLS-DA models allowed us to increase the separation between sample classes and to extract information on discriminating metabolites.

\section{EXPERIMENTAL SECTION}

\section{Reagents}

Ethyl acetate (EtOAc), methanol $(\mathrm{MeOH})$, and dichloromethane (DCM) used for the extraction procedures were purchased from VWR (Fontenay-sous-Bois, France). LC-MS analyses were performed using LC-MS-grade acetonitrile (ACN) and $\mathrm{MeOH}$ (VWR). Milli-Q water was generated by the Millipore ultrapure water system (Waters-Millipore, Milford, MA). Formic acid of mass spectrometry grade (99\%) was obtained from Sigma-Aldrich (St. Quentin-Fallavier, France). 
Bacterial Strains, Culture Conditions, and Metabolite Extraction

Persicivirga (Nonlabens) mediterranea TC4 and TC7 (TC for Toulon Collection), Pseudoalteromonas lipolytica TC8, and Shewanella sp. TC11 strains were isolated from marine biofilms harvested on artificial surfaces immersed in the Mediterranean Sea (Bay of Toulon, France, $43^{\circ} 06^{\prime} 23^{\prime \prime} \mathrm{N}, 5^{\circ} 57^{\prime} 17^{\prime \prime} \mathrm{E}$ ). ${ }^{16}$ All strains were stored at $-80{ }^{\circ} \mathrm{C}$ in $50 \%$ glycerol medium until use and were grown in Väätänen nine salt solution (VNSS) at $20{ }^{\circ} \mathrm{C}$ on a rotator/shaker $(120 \mathrm{rpm})$ to obtain synchronized bacteria in postexponential phase. A cell suspension was used as starting inoculum to prepare planktonic and sessile cultures. Depending on the experiment, these cultures were performed in two different nutrient media: VNSS or marine broth (MB) (BD, Franklin Lakes, NJ), always at the same temperature of $20{ }^{\circ} \mathrm{C}$. In the case of planktonic cultures, precultured bacteria $(10 \mathrm{~mL})$ were suspended in culture medium $(50 \mathrm{~mL})$ at 0.1 absorbance unit $\left(\mathrm{OD}_{600}\right)$ and placed in $250 \mathrm{~mL}$ Erlenmeyer flasks. Strains were grown in a rotary shaker (120 rpm). Medium turbidity was measured at $600 \mathrm{~nm}$ (Genesys 20 spectrophotometer, Thermo Fisher Scientific, Waltham, MA) every hour for the determination of growth curves before metabolite extraction. Cultures were then extracted according to the $\mathrm{OD}_{600}$ value correlated to the growth curve. For sessile conditions, precultured planktonic cells were suspended in culture medium $(10 \mathrm{~mL})$ at 0.1 absorbance unit $\left(\mathrm{OD}_{600}\right)$ in Petri dishes. After 24 or $48 \mathrm{~h}$ of incubation, the culture medium was removed and biofilms were physically recovered by scraping. The resulting mixture was then extracted.

Metabolite extractions were performed with EtOAc, cold $\mathrm{MeOH}$, or a mixture of cold $\mathrm{MeOH} / \mathrm{DCM}(1: 1 \mathrm{v} / \mathrm{v}) .100 \mathrm{~mL}$ of solvent was added to the bacterial culture. The resulting mixture was shaken for $1 \mathrm{~min}$ and then subjected to ultrasounds for $30 \mathrm{~min}$ at $20{ }^{\circ} \mathrm{C}$. For samples extracted with EtOAc, the organic phase was recovered and concentrated to dryness under reduced pressure. Samples extracted with $\mathrm{MeOH}$ or $\mathrm{MeOH} /$ DCM were dried in vacuo. Dried extracts were then dissolved in $\mathrm{MeOH}$ at a concentration of $15 \mathrm{mg} / \mathrm{mL}$. Samples were transferred to $2 \mathrm{~mL} \mathrm{HPLC}$ vials and stored at $-80{ }^{\circ} \mathrm{C}$ until analysis.

For all experiments, bacterial cultures, extraction, and sample preparation were carried out by the same operator.

\section{Metabolic Fingerprinting by LC-MS}

LC-ESI-IT-MS Analyses. The bacterial extracts were analyzed on an Elite LaChrom (VWR-Hitachi, Fontenay-sousBois, France) chromatographic system coupled to an ion trap mass spectrometer (Esquire 6000, Bruker Daltonics, Wissembourg, France). Chromatographic separation was achieved on an analytical core-shell reversed-phase column $(150 \times 3 \mathrm{~mm}$, $2.6 \mu \mathrm{m}$, Kinetex Phenyl-Hexyl, Phenomenex, Le Pecq, France) equipped with a guard cartridge $(4 \times 3 \mathrm{~mm}$, SecurityGuard Ultra Phenomenex) and maintained at $30{ }^{\circ} \mathrm{C}$. The injected sample volume was $5 \mu \mathrm{L}$. The mobile phase consisted of water (A) and ACN (B) containing both $0.1 \%$ of formic acid. The flow rate was $0.5 \mathrm{~mL} / \mathrm{min}$. The elution gradient started at $20 \%$ B during $5 \mathrm{~min}$, ascended to $100 \% \mathrm{~B}$ in $20 \mathrm{~min}$ with a final isocratic step for $10 \mathrm{~min}$; and then returned to $20 \% \mathrm{~B}$ in $0.1 \mathrm{~min}$ and maintained $9.9 \mathrm{~min}$. The electrospray interface (ESI) parameters were set as follows: nebulizing gas $\left(\mathrm{N}_{2}\right)$ pressure at $40 \mathrm{psi}$, drying gas $\left(\mathrm{N}_{2}\right)$ flow at $8 \mathrm{~L} / \mathrm{min}$, drying temperature at $350{ }^{\circ} \mathrm{C}$, and capillary voltage at $4000 \mathrm{~V}$. Mass spectra were acquired in the full scan range $\mathrm{m} / z 50$ to 1200 in positive mode as this mode provides a higher number of metabolite features after filtering and also a better discrimination between clusters in the multivariate statistics. Data were handled with Data Analysis (version 4.3, Bruker Daltonics).

UPLC-ESI-QToF-MS Analyses. The UPLC-MS instrumentation consisted of a Dionex Ultimate 3000 Rapid Separation (Thermo Fisher Scientific) chromatographic system coupled to a QToF Impact II mass spectrometer (Bruker Daltonics). The analyses were performed using an analytical core-shell reversed-phase column $(150 \times 2.1 \mathrm{~mm}, 1.7 \mu \mathrm{m}$, Kinetex Phenyl-Hexyl with a SecurityGuard cartridge, Phenomenex) with a column temperature of $40{ }^{\circ} \mathrm{C}$ and a flow rate of $0.5 \mathrm{~mL} / \mathrm{min}$. The injection volume was $5 \mu \mathrm{L}$. Mobile phases were water (A) and $\mathrm{ACN}(\mathrm{B})$ containing each $0.1 \%(\mathrm{v} / \mathrm{v})$ of formic acid. The elution gradient $(\mathrm{A}: \mathrm{B}, \mathrm{v} / \mathrm{v})$ was as follows: 80:20 from 0 to $1 \mathrm{~min}, 0: 100$ in $7 \mathrm{~min}$ and kept $4 \mathrm{~min}$, and then $80: 20$ at $11.5 \mathrm{~min}$ and kept $2 \mathrm{~min}$. The capillary voltage was set at $4500 \mathrm{~V}$ (positive mode), and the nebulizing parameters were set as follows: nebulizing gas $\left(\mathrm{N}_{2}\right)$ pressure at 0.4 bar, drying gas $\left(\mathrm{N}_{2}\right)$ flow at $4 \mathrm{~L} / \mathrm{min}$, and drying temperature at $180{ }^{\circ} \mathrm{C}$. Mass spectra were recorded from $\mathrm{m} / z 50$ to 1200 at a mass resolving power of 25000 full width at half-maximum (fwhm, $m / z=200)$ and a frequency of $2 \mathrm{~Hz}$. Tandem mass spectrometry analyses were performed thanks to a collisioninduced dissociation (CID) with a collision energy of $25 \mathrm{eV}$. A solution of formate/acetate forming clusters was automatically injected before each sample for internal mass calibration, and the mass spectrometer was calibrated with the same solution before each sequence of samples. Data handling was done using Data Analysis (version 4.3).

Quality Control. For each sequence, a pool sample was prepared by combining $100 \mu \mathrm{L}$ of each bacterial extract. The pool sample was divided into several HPLC vials that were used as quality-control samples (QCs). Samples of each condition were randomly injected to avoid any possible time-dependent changes in LC-MS chromatographic fingerprints. To ensure analytical repeatability, the QCs were injected at the beginning, at the end, and every four samples within each sequence run. Cell-free control samples (media blanks) were prepared in the same way as cultures with cells, and they were randomly injected within the sequence. These blanks allowed the subsequent subtraction of contaminants or components coming from the growth media. Moreover, to assess sample carry-over of the analytical process, three solvent blanks were injected for each set of experiments before the first $\mathrm{QC}$ and after the last QC.

Data Preprocessing and Filtering. LC-MS raw data were converted into netCDF files with a script developed within the Data Analysis software and preprocessed with the XCMS software (version 1.38.0) under R 3.1.0 environment. Peak picking was performed with the "matchedFilter" method for HPLC-IT-MS data and "centwave" method for UPLCQToF-MS data. The other XCMS parameters were as follows: "snthresh" = 5, retention time correction with the obiwarp method ("profstep" $=0.1$ ), peak grouping with " $b w$ " $=5$ for ion trap data, " $b w "=2$ for QToF data and "mzwidth" $=0.5$ for ion trap data, and " $m z$ width" $=0.015$ for QToF data, gap filling with default parameters. ${ }^{19}$

To ensure data quality and remove redundant signals, three successive filtering steps were applied to preprocessed data using an in-house script on $\mathrm{R}$. The first was based on the signal/noise $(\mathrm{S} / \mathrm{N})$ ratio to remove signals observed in medium blanks ( $\mathrm{S} / \mathrm{N}$ set at 10 for features matching between samples 
and medium blanks). The second allowed suppression of signals based on the value of the coefficient of variation $(\mathrm{CV})$ of the intensity of the variables in the QCs (cutoff set at 20\%). A third filtering step was applied using the coefficient of the autocorrelation (with a cutoff set at $80 \%$ ) between variables with a same retention time in the extract samples.

MS/MS Networking. The molecular network was generated on the Internet platform GNPS (http://gnps.ucsd. edu) from MS/MS spectra. Raw data were converted into .mzXML format with DataAnalysis. Data were filtered by removing MS/MS peaks within $\pm 17 \mathrm{Da}$ of the $\mathrm{m} / z$ of the precursor ions. Only the top 6 peaks were conserved in a window of $50 \mathrm{Da}$. Data were clustered using MS-Cluster with a tolerance of $1 \mathrm{Da}$ for precursor ions and of $0.5 \mathrm{Da}$ for MS/MS fragment ions to create a consensus spectrum. Consensus spectra containing fewer than two spectra were eliminated. The resulting spectra were compared with those of the GNPS spectral bank. The molecular network was then generated and previewed directly on GNPS online. Data were imported and treated offline with Cystoscape (version 3.4.0). MS/MS spectra with a high spectral similarity (cosine score $(\mathrm{CS})>0.65$ ) were represented as nodes. Connections between these nodes appeared because the CS was above 0.65 and at least four common ions were detected. The thickness of the connections was proportional to the CS.

Annotation of Biomarkers. Variables of importance were identified from the multivariate statistical analyses (see the Statistical Analyses section). They were then subjected to annotation by searching the most probable molecular formula with the "smartformula" package of DataAnalysis and by analyzing their accurate masses and their fragmentation patterns in comparison with the literature data. Other data available online in KEGG (www.genome.jp/kegg), PubChem (https://pubchem.ncbi.nlm.nih.gov), ChemSpider (www. chemspider.com), Lipid Maps (http://www.lipidmaps.org), Metlin (https://metlin.scripps.edu/), and GNPS (http:// gnps.ucsd.edu) were also used for complementary information.

Statistical Analyses. Simca 13.0.3 software (Umetrics, Umeå, Sweden) was used for all multivariate data analyses and modeling. Data were log10-transformed and mean-centered. Models were built on principal component analysis (PCA) or on partial least-squares discriminant analysis (PLS-DA). PLSDA allowed the determination of discriminating metabolites using the variable importance on projection (VIP). The VIP score value indicates the contribution of a variable to the discrimination between all of the classes of samples. Mathematically, these scores are calculated for each variable as a weighted sum of squares of PLS weights. The mean VIP value is one, and usually VIP values over one are considered as significant. A high score is in agreement with a strong discriminatory ability and thus constitutes a criterion for the selection of biomarkers. All of the models evaluated were tested for over fitting with methods of permutation tests and cross-validation analysis of variance (CV-ANOVA). The descriptive performance of the models was determined by $R^{2} X$ (cumulative) (perfect model: $R^{2} X($ cum $\left.)=1\right)$ and $R^{2} Y$ (cumulative) (perfect model: $R^{2} Y$ $($ cum $)=1)$ values, while their prediction performance was measured by $Q^{2}$ (cumulative) (perfect model: $Q^{2}($ cum $\left.)=1\right), p$ (CV-ANOVA) (perfect model: $p=0$ ) values, and a permutation test $(n=150)$. The permuted model should not be able to predict classes: $R^{2}$ and $Q^{2}$ values at the $Y$-axis intercept must be lower than those of $Q^{2}$ and the $R^{2}$ of the nonpermuted model.
Data Visualization. The heatmap representation was obtained with the PermutMatrix software. ${ }^{20}$ Dissimilarity was calculated with the squared Pearson correlation distance, while the Ward's minimum variance method was used to obtain the hierarchical clustering.

\section{RESULTS AND DISCUSSION}

\section{Selection of the Metabolite Extraction Method (Experiment \#1)}

Metabolomics allows the analysis of many metabolites simultaneously detected in a biological sample. To ensure that the resulting metabolomic profiles characterize the widest range of metabolites of high relevance, the metabolite extraction protocol must be nonselective and highly reproducible. ${ }^{21}$ Therefore, the biological material must be studied after simple preparation steps to prevent any potential degradation or loss of metabolites. In microbial metabolomics, the first step of the sample preparation corresponds to quenching to avoid alterations of the intracellular metabolome, which is known for its fast turnover. ${ }^{22}$ In this study, the first objective was therefore to develop an extraction protocol for LC-MS-based metabolome profiling (exo- and endometabolomes) of marine bacteria that should be applied to any strain, cultivated either planktonically or under sessile conditions, but also easily transposable to natural complex biofilms. For this purpose, liquid-liquid extraction was selected because this process allows quenching and extraction of the bacterial culture in a single step. The second issue is linked to the high salinity of the extracts, which implies a required desalting step. For these reasons, EtOAc, $\mathrm{MeOH}$, and $\mathrm{MeOH} / \mathrm{DCM}$ (1:1) were selected as extractive solvents for this experiment.

For the first experiment, common cultures conditions were selected. Thus the four bacterial strains were grown planktonically in single-species cultures (in VNSS medium), each in biological triplicates, and extracted until they reached their stationary phase (at $t=t_{5}$, Supporting Information Figure S1) and before the decline phase. For each sample, the whole culture was extracted using a predefined set of experimental conditions and analyzed by LC- $(+)-E S I-I T-M S$. The selection of the optimal solvents was performed based on: (i) the number of features detected on LC-MS profiles after filtering, (ii) the ability to discriminate the bacterial strains by multivariate analyses, and (iii) the ease of implementation of the experimental protocol.

In such a rich culture medium, data filtering constitutes a key requirement because bacterial metabolites are masked by components of the culture broth (e.g., peptone, starch, yeast extract). Moreover, such a process was essential to reduce falsepositives and redundant data for the further statistical analyses. First, for each solvent, treatment of all chromatograms with the XCMS package gave a primary data set with $3190 \pm 109$ metabolite features (Supporting Information Figure S2). A primary filtering between variables present in both bacterial extracts and blank samples removed $>80 \%$ of the detected features, which were inferred to culture medium components, solvent contamination, or instrumentation noise. After two additional filtering steps, one with the $\mathrm{CV}$ of variable intensities and the other with the coefficient of autocorrelation across samples and between variables with a same retention time, a final list of $155 \pm 22 \mathrm{~m} / z$ features was reached.

The resulting data showed a different number of detected metabolite features depending on the extraction solvent 

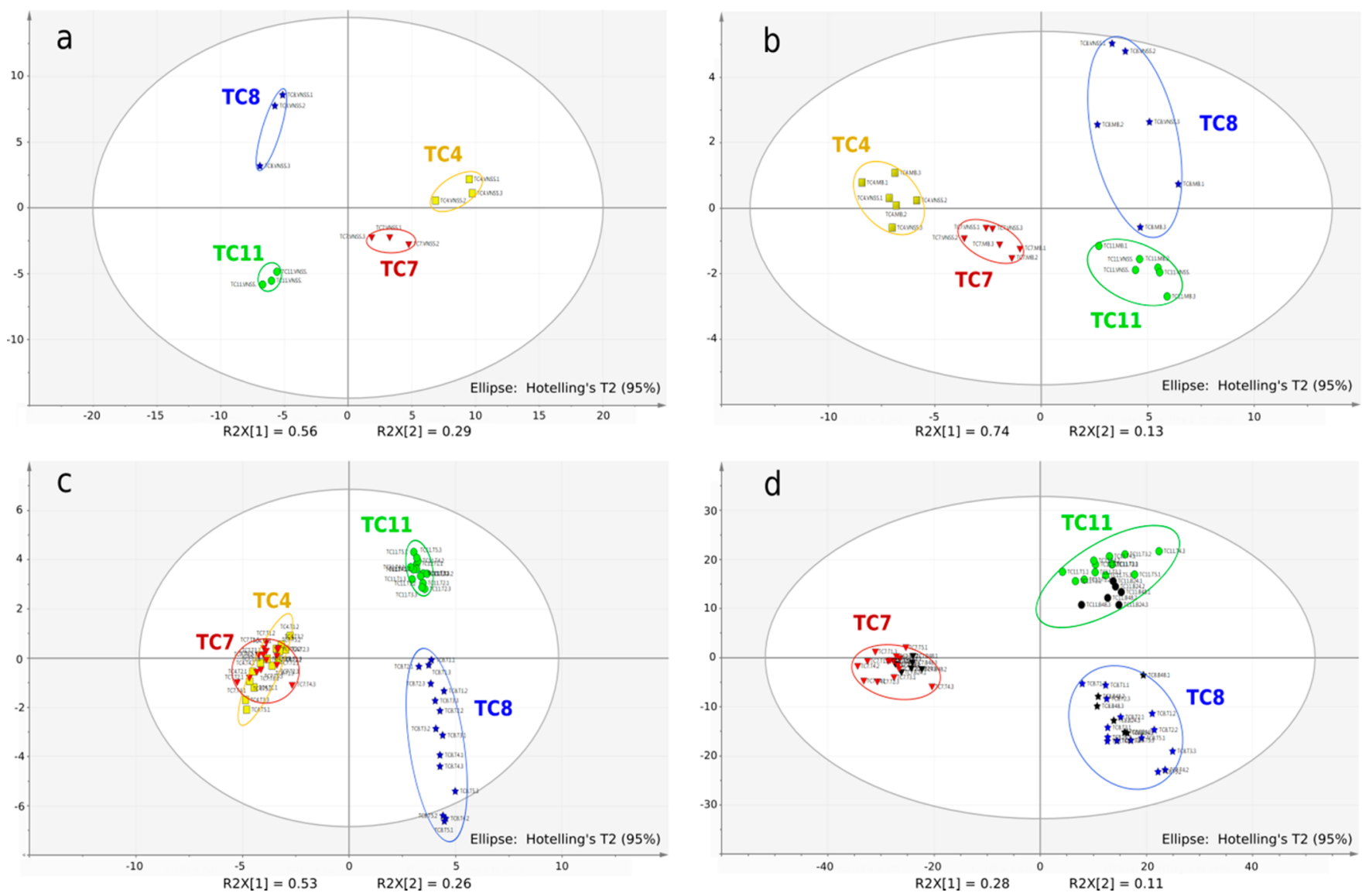

Figure 2. (a) PCA score plot obtained from LC-LRMS profiles of the four bacterial strains (extraction with EtOAc, stationary phase, planktonic cultures in VNSS). (b) PLS-DA score plot obtained from LC-MS profiles of the four bacterial strains (extraction with EtOAc, stationary phase) cultured planktonically in two media (VNSS and MB). (c) PLS-DA score plot obtained from LC-MS profiles of the four bacterial strains (extraction with EtOAc, planktonic cultures in VNSS) at five time points of their growth curve. (d) PLS-DA score plot obtained from LC-MS profiles of three of the strains (extraction with EtOAc) cultured in biofilms (two time points; dark symbols) and planktonic conditions (five time points; colored symbols) in VNSS.

Table 1. Summary of the Parameters for the Assessment of the Quality and of the Validity of the PLS-DA Models Used for the Discrimination of the Bacterial Strains According to Different Culture or Analysis Conditions

\begin{tabular}{|c|c|c|c|c|c|c|c|c|c|}
\hline experiments & parameters & models & $N^{\circ a}$ & $R^{2} X_{\text {cum }}{ }^{b}$ & $R^{2} Y_{\text {cum }}{ }^{c}$ & $Q^{2} Y_{\text {cum }}{ }^{d}$ & $R$ intercept $^{e}$ & $Q$ intercept $^{e}$ & $P^{f}$ \\
\hline \multirow{6}{*}{$\begin{array}{l}\text { extraction solvents (VNSS, planktonic, stationary } \\
\text { phase) }\end{array}$} & \multirow[t]{2}{*}{ EtOAc } & 4 classes $^{g}$ & 5 & 0.951 & 0.985 & 0.880 & 0.660 & -0.350 & 0.07 \\
\hline & & 3 classes $^{h}$ & 3 & 0.896 & 0.974 & 0.939 & 0.326 & -0.377 & 0.0001 \\
\hline & \multirow[t]{2}{*}{$\mathrm{MeOH}$} & 4 classes $^{g}$ & 5 & 0.957 & 0.995 & 0.967 & 0.637 & -0.460 & 0.07 \\
\hline & & 3 classes $^{h}$ & 3 & 0.886 & 0.985 & 0.935 & 0.399 & -0.373 & $<0.0001$ \\
\hline & \multirow[t]{2}{*}{$\mathrm{MeOH} / \mathrm{DCM}$} & 4 classes $^{g}$ & 6 & 0.966 & 0.997 & 0.820 & 0.861 & -0.070 & 1 \\
\hline & & 3 classes $^{h}$ & 4 & 0.930 & 0.991 & 0.956 & 0.656 & -0.321 & 0.001 \\
\hline \multirow[t]{4}{*}{ culture media (EtOAc, planktonic, stationary phase) } & \multirow[t]{2}{*}{ MB } & 4 classes $^{g}$ & 7 & 0.978 & 0.987 & 0.852 & 0.871 & -0.051 & 0.87 \\
\hline & & 3 classes $^{h}$ & 3 & 0.916 & 0.910 & 0.800 & 0.316 & -0.413 & 0.007 \\
\hline & \multirow[t]{2}{*}{ VNSS \& MB } & 4 classes $^{g}$ & 5 & 0.955 & 0.918 & 0.819 & 0.344 & -0.469 & $<0.0001$ \\
\hline & & 3 classes $^{h}$ & 4 & 0.941 & 0.931 & 0.877 & 0.294 & -0.402 & $<0.0001$ \\
\hline \multirow[t]{2}{*}{ growth phases (EtOAc, VNSS, planktonic) } & \multirow{2}{*}{$\begin{array}{l}\text { all growth } \\
\text { phases }\end{array}$} & 4 classes $^{g}$ & 6 & 0.640 & 0.970 & 0.929 & 0.570 & -0.324 & 0 \\
\hline & & 3 classes $^{h}$ & 4 & 0.579 & 0.975 & 0.957 & 0.578 & -0.322 & 0 \\
\hline \multirow[t]{2}{*}{ culture modes (EtOAc, VNSS, stationary phase $)^{j}$} & biofilm & 3 classes $^{i}$ & 3 & 0.638 & 0.994 & 0.984 & 0.580 & -0.245 & $<0.0001$ \\
\hline & $\begin{array}{l}\text { biofilm \& } \\
\text { planktonic }\end{array}$ & 3 classes $^{i}$ & 4 & 0.543 & 0.991 & 0.977 & 0.618 & -0.136 & 0 \\
\hline \multirow{2}{*}{$\begin{array}{l}\text { platforms comparison (EtOAc, VNSS, planktonic, } \\
\text { stationary phase) }\end{array}$} & \multirow[t]{2}{*}{ HRMS } & 4 classes $^{g}$ & 5 & 0.867 & 0.989 & 0.785 & 0.661 & -0.440 & 0.56 \\
\hline & & 3 classes $^{h}$ & 3 & 0.735 & 0.989 & 0.939 & 0.489 & -0.411 & 0.0001 \\
\hline
\end{tabular}

${ }^{a}$ Number of components of the model. ${ }^{b}$ Cumulative modeled variation in $X$ matrix. ${ }^{c}$ Cumulative modeled variation in $Y$ matrix. ${ }^{d}$ Cumulative predicted variation in $Y$ matrix. ${ }^{e} R$ and $Q$ intercepts obtained after permutation test $(n=150) .{ }^{f} p$ value obtained from 7-fold cross validation ANOVA of PLS-DA. ${ }^{g}$ One class for each strain. ${ }^{h}$ TC4 and TC7 strains gathered in a same class. ${ }^{i}$ One class for each strain. ${ }^{j}$ Only three strains (TC7, TC8, and TC11) were used for this experiment. 
(Supporting Information Figure S3): $\mathrm{MeOH} / \mathrm{DCM}$ yielded a higher number of metabolites for TC4 and TC11, while EtOAc was the most effective extraction solvent for TC7 and TC8. This result was expected because previous works showed that the extraction method had a strong effect on the detected microbial metabolome, with the physicochemical properties of the extraction solvent being one of the main factor of the observed discrepancies. ${ }^{23,24}$ The extraction parameters had an effect not only on the number of detected features but also on their concentration. ${ }^{25}$ The LC-MS data sets were analyzed by PCA and PLS-DA to evaluate the potential of the method to discriminate among the bacterial strains according to the extraction solvent system. PCA evidenced interstrain cleavage on the score plots (Figure $2 \mathrm{a}$ and Supporting Information Figure S4a,b). For each solvent, samples from TC4 and TC7, on one hand, and from TC8 and TC11, on the other hand, were clearly distinguished on the first component, which accounted for $56-72 \%$ of the total variance. The second component, with 12 to $29 \%$, allowed the distinction between TC8 and TC11 and, to a lesser extent, between TC4 and TC7. To find discriminating biomarkers, PLS-DA was also applied to the LC-MS data (one model by solvent condition and one class by strain). For each extraction solvent, the resulting score plots showed three distinct clusters composed of both $P$. mediterranea strains (TC4 and TC7), TC8 and TC11, respectively (data not shown). The PLS-DA four-class models gave $R^{2} X$ cum and $R^{2} Y$ cum values of $0.951-0.966$ and $0.985-$ 0.997, respectively, showing the consistency of the obtained data, and $Q^{2} Y$ cum values of $0.820-0.967$, estimating their predictive ability (Table 1$)$. Nevertheless, the $p$ values $(>0.05)$ obtained from the cross validation indicated that the bacterial samples were not significantly separated according to the strain, while the $R$ intercept values $(>0.4)$ obtained from a permutation test $(n=150)$ showed overfitting of the models. Taking these results into account, three-class PLS-DA models regrouping the TC4 and TC7 strains into a same class were constructed. The resulting $R^{2} X$ cum $(0.886-0.930), R^{2} Y$ cum (0.974-0.991), and $Q^{2} Y$ cum (0.935-0.956) values attested the quality of these improved models. In addition, a permutation test $(n=150)$ allowed the successful validation of the PLS-DA models: $R$ intercept values $(<0.4$, except for $\mathrm{MeOH} / \mathrm{DCM})$ and $Q$ intercept values $(<-0.2)$ indicated that no overfitting was observed, while $p$ values $(<0.05)$ showed that the three groups fitted by the models were significantly different (Table 1, Supporting Information Figure S4c-e). Samples extracted with $\mathrm{MeOH}$ and EtOAc showed higher quality and more robust PLS-DA models for the strain discrimination than those obtained after extraction with $\mathrm{MeOH} / \mathrm{DCM}$. For all of these reasons, EtOAc was selected for metabolome extraction in the subsequent experiments. These results were in accordance with the use of a similar protocol in recent studies dealing with the chemical profiling of marine bacteria. ${ }^{26-28}$ Three culture parameters (culture media, phase of growth, and mode of culture) were then analyzed sequentially to evaluate their respective impact on the interstrain metabolic discrimination.

Impact of the Culture Medium (Experiment \#2)

The influence of the culture medium on the marine bacteria metabolome has been poorly investigated..$^{15,25,27,29}$ To ascertain that the chemical discrimination of the bacterial strains studied was not medium-dependent, a second culture broth was used. This second set of experiments was designed as follows: the four bacterial strains were cultivated in parallel in VNSS and
MB media (each in biological triplicates) until they reached the stationary phase $\left(t=t_{5}\right.$, Supporting Information Figure S1), and their organic extracts (extraction with EtOAc) were analyzed by LC-MS. Just like in the case of VNSS, MB is a salt-rich medium widely used for marine bacterial cultures. The number of metabolites detected and the chemical discrimination between the bacterial strains were then determined for this set of samples. First, the number of metabolites obtained after the three filtering steps was similar for both culture media (Supporting Information Figure S5), and all of the detected $\mathrm{m} / z$ features were common to both media. This result showed the robustness of the filtering method because the chemical compositions of both culture media are highly different (Supporting Information Table S1). Indeed, MB contains more salts, and, in terms of organic components, higher amounts of yeast extract and peptone, while starch and glucose are specific ingredients of VNSS. A small difference was observed on the PCA score plots obtained with samples from a single strain cultured in these two different media, but the low number of samples did not allow the validation of the corresponding PLS-DA models (data not shown). Whatever the medium, an obvious clustering pattern for each of the four strains was observed on the PCA score plots when all of the samples were considered (Supporting Information Figure S6a). Four- and three-class PLS-DA models were constructed to evaluate the discrimination capacity of the method. As demonstrated for VNSS cultures (Supporting Information Figure S4c), the PLS-DA three-class model obtained with the bacteria grown in MB (Table 1 and Supporting Information Figure S6b) also showed a clear separation between the groups $(p<0.05)$, and it was statistically validated by a permutation test. When the whole data set (VNSS and MB) was analyzed, the resulting PLS-DA models (Table 1), which passed crossvalidation and permutation test, indicated that the bacterial samples could be efficiently discriminated at the species level. On the basis of the PLS-DA score plot, TC8 was the bacterial strain, which demonstrated the higher metabolic variation with the culture media used (Figure $2 \mathrm{~b}$ ). It is now well established that changing bacterial culture media not only affects the metabolome quantitatively but also has a significant impact on the expression of some distinct biosynthetic pathways. Such an approach, named OSMAC (One Strain-MAny Compounds), has been used in recent years to improve the number of secondary metabolites produced by a single microbial strain. ${ }^{30}$ In the present study, some intrastrain differences were observed between cultures in both media, but they did not prevent from a clear interstrain discrimination. Therefore, these results showed that this method allowed the discrimination between samples of the three marine biofilm-forming bacterial species, even if they are grown in distinct media.

\section{Impact of the Growth Phase (Experiment \#3)}

Growth of bacteria in suspension, as planktonic microorganisms, follows a typical curve with a sequence of a lag phase, an exponential phase (multiplication of cells), a stationary phase (stabilization), and a decline phase. To date, only a few studies have focused on differences in the metabolome of microorganisms along their growth phase, ${ }^{13,31,32}$ and most of these analyses were performed by NMR and GC-MS. These different culture phases are related to the rapid bacterial response to environmental changes and thus to different metabolic expressions. To determine the impact of this biological variation on the discrimination 
between bacterial cell samples, the metabolome content was analyzed (in biological triplicates) for the four strains grown in VNSS at five times of their different growth stages: two time points during the exponential phase $\left(t_{1}\right.$ and $\left.t_{2}\right)$, one at the end of the exponential phase $\left(t_{3}\right)$, and two others during the stationary phase $\left(t_{4}\right.$ and $t_{5}$ ) (Supporting Information Figure $S 1)$. All aliquots were treated with the selected extraction protocol, followed by analysis with LC-MS. The data obtained for the four strains were preprocessed, filtered, and then analyzed by PCA and PLS-DA. As shown in Figure S1, the strains grew differently, as indicated by $\mathrm{OD}_{600}$ changes: the exponential phase of all of the strains started directly after inoculation and occurred during $3 \mathrm{~h}$ for TC8 and TC11 and $8 \mathrm{~h}$ for TC4 and TC7, respectively. After filtering, the data showed that among all of the strains TC8 produced the highest number of metabolites in all phases, while TC7 was always the less productive. The number of metabolites detected was higher for TC8 during the stationary phase, while it was slightly higher for TC11 during the exponential phase, and no significant differences were noticed for TC4 and for TC7 (Supporting Information Figure S7). For each strain, most of the detected $\mathrm{m} / z$ signals were found in both growth phases, but more than two-thirds of them were present in higher amounts during the stationary phase.

To determine if this method was also able to differentiate between the phases of growth, PLS-DA models were then constructed for each bacterial strain with the LC-MS profiles (Supporting Information Table S2). For TC8, bacterial cultures were clearly discriminated with their growth phase, as described on the corresponding PLS-DA score plot (Supporting Information Figure S8a). This constructed PLS-DA model was well-fitted to the experimental data: It consisted of four components, and the two first explained almost $75 \%$ of the variation. The first dimension showed a significant separation between cultures harvested at the beginning and the middle of the exponential phase $\left(t_{1}\right.$ and $\left.t_{2}\right)$, the end of this same growth phase $\left(t_{3}\right)$, and the stationary phase $\left(t_{4}\right.$ and $\left.t_{5}\right)$, while the second one emphasized the discrimination of cultures collected at the end of the exponential phase $\left(t_{3}\right)$ from the others. With a less pronounced separation between samples of the exponential phase, a similar pattern was observed for TC4 and, to a lesser extent, for TC11 (Supporting Information Figure S8b,c). For TC7, no PLS-DA model allowed highlighting significant differences between samples with the growth phase (data not shown). Finally, the discrimination between all of the bacterial species harvested during the two growth phases (five time points) was analyzed. The resulting PLS-DA model explained $>78 \%$ of the variance of the data set (Table 1 and Figure $2 \mathrm{c}$ ). Here again, the metabolome of the TC8 strain showed the most important variability, but a clear discrimination between the metabolomes of the four bacterial strains was observed whatever the phase of growth. In accordance with their taxonomic proximity, it was highlighted that both $P$. mediterranea strains (TC4 and TC7) were closely related.

It is now well-established that drastic changes may occur in bacterial metabolic production at the transition from exponential phase to stationary phase. This phenomenon is often due to a lowered protein biosynthesis, which induces the biosynthetic machinery to switch from a metabolic production mainly dedicated to cell growth during exponential phase toward alternative metabolism, producing a new set of compounds during the stationary phase. ${ }^{3,34}$ However, in contrast with well-studied model microorganisms, several marine bacteria undergo a stand-by step between these two growth phases. ${ }^{35,36}$ For each strain, our results showed that most of the changes between the growth phases correspond to the upregulation of a large part of the metabolites during the stationary phase. This trend was already observed in previous studies, ${ }^{31,32}$ but opposite results have also been described due to distinct metabolome coverage or studied microorganism. ${ }^{13}$ These bibliographic data were also in accordance with the different behavior of each of the four strains when the metabolome, restricted to the extraction and analytical procedures, was investigated at different time points of the growth curve. The chemical discrimination of these bacteria was thus not dependent on their growth phase. Overall, because the chemical diversity seemed to be higher during the stationary phase, this growth phase was then chosen for the rest of the study.

\section{Impact of the Mode of Culture (Experiment \#4)}

The bacterial strains were isolated from marine biofilms developed on artificial surfaces immersed in situ. ${ }^{16}$ In addition to their facility to grow in vitro, these strains were chosen for their propensity to form biofilms. The intrinsic differences between the metabolisms of planktonic and biofilm cells and the impact of these two modes of culture on the interstrain discrimination were analyzed by LC-MS profiling of three of the bacteria. Indeed, due to the chemical similarity of both $P$. mediterranea strains, only TC4 was used for this experiment. For this purpose, these strains were cultured in triplicate in planktonic (at five points of their growth curve) and biofilm modes (at two culture times: 24 and $48 \mathrm{~h}$ ). This difference in growth time between both culture modes was due to the slowgrowing nature of biofilms. To compare accurately the two modes of culture, the development of biofilms was performed under static conditions and in the same medium as those used for planktonic growth (VNSS). For each strain, PLS-DA models were constructed and showed a clear discrimination between samples with their culture mode with total variances ranging from 52 to $59 \%$ (Supporting Information Figure S9). PLS-DA models with good-quality parameters were obtained, and validation values indicated that they could be regarded as predictable (Supporting Information Table S2). Moreover, a similar number of $\mathrm{m} / z$ features upregulated specifically in one of the two culture modes was detected for each strain. When dealing with the interstrain discrimination for bacteria cultured as biofilms, the three strains were clearly separated on the PCA score plot, and the total variance due to the two main projections accounted for 59\% (Supporting Information Figure S10a). The corresponding PLS-DA model showed a similar trend and gave good results, indicating that this model could distinguish the three strains (Table 1 and Supporting Information Figure S10b). When the full data set (biofilms and planktonic cultures) was analyzed, the same pattern was further noticed with the occurrence of one cluster by strain on the PCA score plot (Supporting Information Figure S11). A PLS-DA model was built and demonstrated again, after validation, a good separation among all of the strains (Table 1 and Figure $2 \mathrm{~d}$ ).

To date, the few metabolomics studies undertaken on biofilms were mostly based on NMR, which is limited by intrinsic low sensitivity. ${ }^{37,38}$ More specifically, only two studies have used a metabolomic approach with the aim of analyzing marine bacterial biofilms. ${ }^{39,40}$ It is now well-established that in many aquatic environments most of the bacteria are organized 


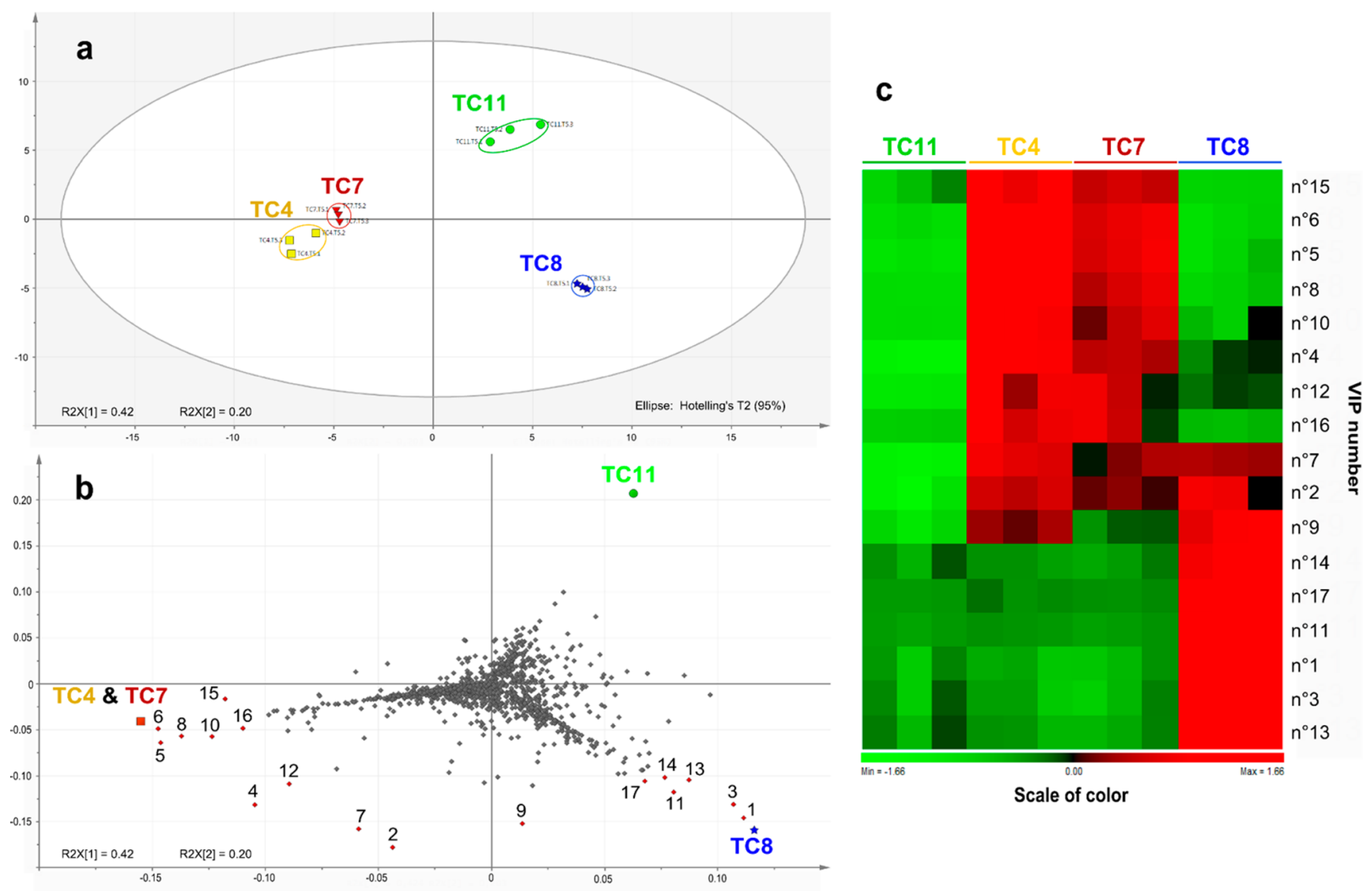

Figure 3. (a) PLS-DA score plot obtained from LC-HRMS profiles of the four bacterial strains (extraction with EtOAc, stationary phase, planktonic cultures in VNSS). (b) PLS-DA loading plots with the most contributing mass peaks (VIPs) numbered from 1 to 17. (c) Heatmap of the 17 differential metabolites with VIP values $\geq 3.0$ from the PLS-DA model. Detailed VIPs description is given in Table 2.

in biofilms, and this living mode is significantly different from its planktonic counterpart. ${ }^{41}$ Deep modifications occur in bacterial cells at various levels (e.g., gene expression, proteome, transcriptome) during the transition from free-living planktonic to biofilm states. ${ }^{42}$ Biofilm cells have traditionally been described as metabolically dormant with reduced growth and metabolic activity. Additionally, cells in biofilms show a higher tolerance to stress (e.g., chemical agents, competition, and predation). On the basis of these data, a liquid culture alone does not allow a full understanding of the ecological behavior or the realistic response to a specific challenge in the case of benthic marine bacteria. For the TC4, TC8, and TC11 strains, PLS-DA models allowed an unambiguous distinction between biofilm and planktonic samples at different ages. As described in the literature for other bacteria, these results agreed with a significant metabolic shift between the two modes of culture whatever the strain and the culture time. Considering the biofilm samples and the whole set of samples, our results demonstrated that chemical profiling by LC-MS followed by PLS-DA analysis led to a clear discrimination between the three strains. Therefore, the interstrain metabolic differences are more significant than the intrastrain differences inherent to the culture mode.

\section{Analytical Platforms Comparison and Identification of Putative Biomarkers}

The data collected during the first part of this study did not allow the annotation of the biomarkers. In this last part, both accurate MS and MS/MS data were obtained from a limited pool of samples (four strains, EtOAc as extraction solvent, planktonic cultures in VNSS until the stationary phase) with an UPLC-ESI-QToF equipment. After extraction and filtering, the data obtained from the LC-HRMS profiles were subjected to chemometric analyses. The resulting PCA and PLS-DA score plots (Supporting Information Figure S12 and Figure 3a) were compared with those obtained with the same set of samples on the previous LC-LRMS platform (Figure 2a and Supporting Information Figure S4c). For both platforms, the PCA score plots exhibited a clear discrimination between the four strains with a separation of the two couples TC4/TC7 and TC8/ TC11 on the first component and between the strains of each pair on the second component. The main difference relies in the total variance accounted for by these two first components, which was lower in the case of the HRMS platform (64\% instead of $85 \%$ for the LRMS platform). These results prove the robustness of the method.

The subsequent step was to build a supervised discrimination model using PLS-DA for UPLC-QToF data. As already described for HPLC-IT-MS data, the resulting three-class PLS-DA model led to a proper differentiation of the three bacterial groups (Table 1). Moreover, despite the different kind of chromatographic conditions (HPLC vs UPLC) and mass spectrometry instrumentations (ESI-IT vs ESI-QToF), the two platforms gave similar results and the same conclusion was made with other sets of samples. Moreover, samples used for the study of the impact of the growth phase and the mode of culture on the TC8 strain were also analyzed on the HRMS 

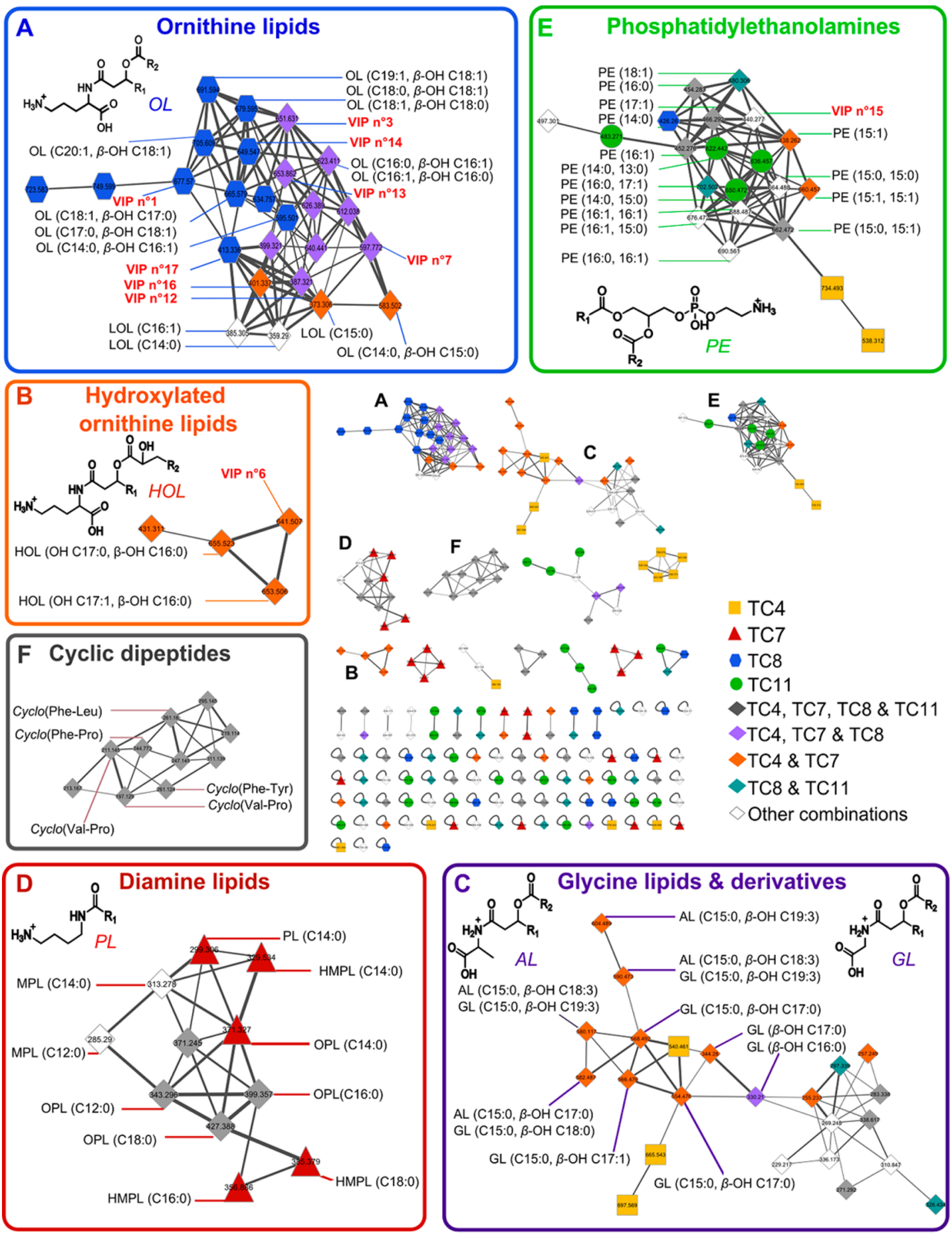

Figure 4. Molecular networks of HRMS fragmentation data obtained from cultures of the four bacterial strains (extraction with EtOAc, stationary phase, planktonic cultures in VNSS). AL: alanine lipid, GL: glycine lipid, HOL: hydroxylated ornithine lipid, HMPL: hydroxylated and methylated putrescine lipid, LOL: lyso-ornithine lipid. MPL: methylated putrescine lipid, OL: ornithine lipid, OPL: oxidized putrescine lipid, PE: phosphatidylethanolamine, PL: putrescine lipid.

platform, and the resulting PLS-DA model was similar to those obtained on the LC-LRMS platform (Supporting Information Figures S8a and S13).

In a second step, the aim was to identify putative biomarkers for each bacterial strain. Metabolome annotation is often considered as a bottleneck in the metabolomics data analysis, which is even more challenging for nonstudied species. For this reason, a molecular network was constructed based on MS/MS data (Figure 4). This analysis has the main advantage to organize mass spectra by fragmentation similarity, rendering easier the annotation of compounds of a same chemical family. ${ }^{43}$ The molecular network constructed with a set of data including all of the strains (EtOAc as extraction solvent, planktonic cultures in VNSS until the stationary phase) highlighted several clusters. At the same time, the most discriminating $\mathrm{m} / \mathrm{z}$ features in the PLS-DA model (Figure $3 \mathrm{~b}, \mathrm{c}$ ) were selected based on their VIP score, which resulted in 17 compounds with VIP value equal to or higher than 3 (Table 2). 
Table 2. List of the Biomarkers (VIP Value $\geq 3$ ) Identified by LC-HRMS for the Discrimination of the Four Bacterial Strains

\begin{tabular}{|c|c|c|c|c|c|c|c|c|c|}
\hline $\begin{array}{l}\text { VIP } \\
\text { number }\end{array}$ & $m / z$ & $\begin{array}{l}\mathrm{RT} \\
(\mathrm{s})\end{array}$ & $\begin{array}{l}\text { VIP } \\
\text { value }\end{array}$ & formula & $\begin{array}{l}\text { mass } \\
\text { error } \\
(\mathrm{ppm})\end{array}$ & $m \sigma^{a}$ & $\begin{array}{l}I \operatorname{expl}^{b} \\
(\%)^{b}\end{array}$ & MS/MS fragment ions (relative abundance in \%) & $\begin{array}{c}\text { putative } \\
\text { identification }^{c}\end{array}$ \\
\hline 1 & 677.5806 & 438 & 4.0 & $\mathrm{C}_{41} \mathrm{H}_{77} \mathrm{~N}_{2} \mathrm{O}_{5}$ & 3.8 & 4.9 & 63.5 & $\begin{array}{l}659(3)^{d}, 413(16)^{e}, 395(62)^{f}, 377(62)^{g}, 159(4)^{h}, 141 \\
(3)^{h}, 133(5)^{h}, 115(100)^{h}, 70(25)^{h}\end{array}$ & $\begin{array}{l}\text { OL } \\
(\mathrm{C} 18: 1, \mathrm{C} 18: 1)\end{array}$ \\
\hline 2 & 625.5501 & 425 & 4.0 & $\mathrm{C}_{37} \mathrm{H}_{73} \mathrm{~N}_{2} \mathrm{O}_{5}$ & 2.0 & 3.6 & 93.6 & $\begin{array}{l}607(2)^{d}, 387(11)^{e}, 369(41)^{f}, 351(44)^{g}, 159(6)^{h}, 141 \\
(3)^{h}, 133(5)^{h}, 115(100)^{h}, 70(24)^{h}\end{array}$ & $\begin{array}{l}\mathrm{OL} \\
(\mathrm{C} 16: 0, \mathrm{C} 16: 0)\end{array}$ \\
\hline 3 & 651.5653 & 429 & 3.7 & $\mathrm{C}_{39} \mathrm{H}_{75} \mathrm{~N}_{2} \mathrm{O}_{5}$ & 2.7 & 5.9 & 61.7 & $\begin{array}{l}633(3)^{d}, 413(6)^{e}, 395(24)^{f}, 377(24)^{g}, 159(4)^{h}, 141 \\
(3)^{h}, 133(4)^{h}, 115(100)^{h}, 70(21)^{h}\end{array}$ & $\begin{array}{l}\mathrm{OL} \\
(\mathrm{C} 18: 1, \mathrm{C} 16: 0)\end{array}$ \\
\hline 4 & 611.5354 & 426 & 3.7 & $\mathrm{C}_{36} \mathrm{H}_{71} \mathrm{~N}_{2} \mathrm{O}_{5}$ & 0.6 & 2.8 & 62.5 & $\begin{array}{l}593(2)^{d}, 387(12)^{e}, 369(43)^{f}, 351(48)^{g}, 159(4)^{h}, 141 \\
(3)^{h}, 133(4)^{h}, 115(100)^{h}, 70(25)^{h}\end{array}$ & $\begin{array}{l}\mathrm{OL} \\
(\mathrm{C} 16: 0, \mathrm{C} 15: 0)\end{array}$ \\
\hline 5 & 627.5304 & 408 & 3.5 & $\mathrm{C}_{36} \mathrm{H}_{71} \mathrm{~N}_{2} \mathrm{O}_{6}$ & 0.4 & 2.4 & 81.7 & $\begin{array}{l}609(<1)^{d}, 591(<1)^{i}, 387(8)^{f}, 369(58)^{g}, 351(62)^{j} \\
159(7)^{h}, 141(3)^{h}, 133(5)^{h}, 115(100)^{h}, 70(16)^{h}\end{array}$ & $\begin{array}{l}\mathrm{HOL} \\
(\mathrm{C} 16: 0, \mathrm{C} 15: 0)\end{array}$ \\
\hline 6 & 641.5462 & 417 & 3.4 & $\mathrm{C}_{37} \mathrm{H}_{73} \mathrm{~N}_{2} \mathrm{O}_{6}$ & 0.2 & 1.7 & 80.5 & $\begin{array}{l}623(1)^{d}, 605(<1)^{i}, 401(7)^{f}, 383(50)^{g}, 365(52)^{j}, 159 \\
(6)^{h}, 141(3)^{h}, 133(5)^{h}, 115(100)^{h}, 70(15)^{h}\end{array}$ & $\begin{array}{l}\mathrm{HOL} \\
\quad(\mathrm{C} 17: 0, \mathrm{C} 15: 0)\end{array}$ \\
\hline 7 & 597.5184 & 418 & 3.3 & $\mathrm{C}_{35} \mathrm{H}_{69} \mathrm{~N}_{2} \mathrm{O}_{5}$ & 2.8 & 1.8 & 57.7 & $\begin{array}{l}579(2)^{d}, 387(2)^{e}, 369(8)^{f}, 351(10)^{g}, 159(4)^{h}, 141 \\
\quad(3)^{h}, 133(5)^{h}, 115(100)^{h}, 70(36)^{h}\end{array}$ & $\begin{array}{l}\mathrm{OL} \\
(\mathrm{C} 16: 0, \mathrm{C} 14: 0)\end{array}$ \\
\hline 8 & 613.5149 & 398 & 3.3 & $\mathrm{C}_{35} \mathrm{H}_{69} \mathrm{~N}_{2} \mathrm{O}_{6}$ & 0.2 & 1.3 & 78.9 & $\begin{array}{l}595(1)^{d}, 577(<1)^{i}, 387(5)^{f}, 369(33)^{g}, 351(37)^{j}, 159 \\
(7)^{h}, 141(3)^{h}, 133(5)^{h}, 115(100)^{h}, 70(22)^{h}\end{array}$ & $\begin{array}{l}\mathrm{HOL} \\
(\mathrm{C} 16: 0, \mathrm{C} 14: 0)\end{array}$ \\
\hline 9 & 623.5339 & 425 & 3.2 & $\mathrm{C}_{37} \mathrm{H}_{71} \mathrm{~N}_{2} \mathrm{O}_{5}$ & 2.9 & 1.5 & 66.8 & $\begin{array}{l}605(2)^{d}, 385(3)^{e}, 367(12)^{f}, 349(12)^{g}, 159(4)^{h}, 141 \\
(3)^{h}, 133(4)^{h}, 115(100)^{h}, 70(24)^{h}\end{array}$ & OL $(\mathrm{C} 16: 1, \mathrm{C} 16: 0)$ \\
\hline 10 & 639.5299 & 403 & 3.0 & $\mathrm{C}_{37} \mathrm{H}_{71} \mathrm{~N}_{2} \mathrm{O}_{6}$ & 1.2 & 10.5 & 81.0 & $\begin{array}{l}621(1)^{d}, 603(<1)^{i}, 399(<1)^{f}, 381(58)^{g}, 363(56)^{j} \\
159(7)^{h}, 141(3)^{h}, 133(6)^{h}, 115(100)^{h}, 70(21)^{h}\end{array}$ & $\begin{array}{l}\mathrm{HOL} \\
\quad(\mathrm{C} 17: 1, \mathrm{C} 15: 0)\end{array}$ \\
\hline 11 & 621.5182 & 409 & 3.0 & $\mathrm{C}_{37} \mathrm{H}_{69} \mathrm{~N}_{2} \mathrm{O}_{5}$ & 3.0 & 8.6 & 61.0 & $\begin{array}{l}603(2)^{d}, 385(15)^{e}, 367(54)^{f}, 349(52)^{g}, 159(5)^{h}, 141 \\
(3)^{h}, 133(6)^{h}, 115(100)^{h}, 70(36)^{h}\end{array}$ & $\begin{array}{l}\mathrm{OL} \\
(\mathrm{C} 16: 1, \mathrm{C} 16: 1)\end{array}$ \\
\hline 12 & 387.3218 & 294 & 3.0 & $\mathrm{C}_{21} \mathrm{H}_{43} \mathrm{~N}_{2} \mathrm{O}_{4}$ & -0.3 & 12 & 103.6 & $\begin{array}{c}369(3)^{d}, 351(5)^{i}, 159(1)^{h}, 141(3)^{h}, 133(7)^{h}, 115 \\
(100)^{h}, 70(62)^{h}\end{array}$ & LOL (C16:0) \\
\hline 13 & 653.5808 & 446 & 3.0 & $\mathrm{C}_{39} \mathrm{H}_{77} \mathrm{~N}_{2} \mathrm{O}_{5}$ & 2.9 & 7.1 & 63.0 & $\begin{array}{l}635(2)^{d}, 415(8)^{e}, 397(28)^{f}, 379(29)^{g}, 159(5)^{h}, 141 \\
(3)^{h}, 133(5)^{h}, 115(100)^{h}, 70(35)^{h}\end{array}$ & $\begin{array}{l}\text { OL } \\
(\mathrm{C} 18: 0, \mathrm{C} 16: 0)\end{array}$ \\
\hline 14 & 649.5498 & 430 & 3.0 & $\mathrm{C}_{39} \mathrm{H}_{73} \mathrm{~N}_{2} \mathrm{O}_{5}$ & 2.5 & 2.6 & 66.0 & $\begin{array}{l}631(2)^{d}, 413(7)^{e}, 395(27)^{f}, 377(27)^{g}, 159(4)^{h}, 141 \\
(3)^{h}, 133(4)^{h}, 115(100)^{h}, 70(23)^{h}\end{array}$ & $\begin{array}{l}\text { OL } \\
(\mathrm{C} 18: 1, \mathrm{C} 16: 1)\end{array}$ \\
\hline 15 & 440.2769 & 340 & 3.0 & $\mathrm{C}_{20} \mathrm{H}_{43} \mathrm{NO}_{7} \mathrm{P}$ & 0.2 & 15.2 & 76.6 & $299(100)^{k}$ & $\mathrm{PE}(\mathrm{C} 15: 0)$ \\
\hline 16 & 401.3373 & 305 & 3.0 & $\mathrm{C}_{22} \mathrm{H}_{41} \mathrm{~N}_{2} \mathrm{O}_{4}$ & 0.1 & 16.0 & 81.5 & $\begin{array}{l}383(4)^{d}, 365(6)^{i}, 159(2)^{h}, 141(4)^{h}, 133(7)^{h}, 115 \\
(100)^{h}, 70(60)^{h}\end{array}$ & LOL $(\mathrm{C} 17: 0)$ \\
\hline 17 & 413.5187 & 304 & 3.0 & $\mathrm{C}_{23} \mathrm{H}_{45} \mathrm{~N}_{2} \mathrm{O}_{4}$ & 0.2 & 13.6 & 80.5 & $\begin{array}{l}395(4)^{d}, 377(6)^{i}, 159(2)^{h}, 141(4)^{h}, 133(10)^{h}, 115 \\
(100)^{h}, 70(51)^{h}\end{array}$ & LOL (C18:1) \\
\hline
\end{tabular}

${ }^{a}$ Constructor statistical match factor obtained by comparison of the theoretical and observed isotopic pattern. ${ }^{b}$ Total intensity of the explained peaks with respect to the total intensity of all peaks in the fragment spectrum peak list. ${ }^{c} \mathrm{HOL}$ : hydroxylated ornithine lipid, LOL: lyso-ornithine lipid, OL: ornithine lipid, PE: phosphatidylethanolamine. ${ }^{d}\left[\mathrm{M}+\mathrm{H}-\mathrm{H}_{2} \mathrm{O}\right]^{+} .{ }^{e}[\mathrm{M}+\mathrm{H}-\mathrm{RCOH}]^{+} .{ }^{f}\left[\mathrm{M}+\mathrm{H}-\mathrm{H}_{2} \mathrm{O}-\mathrm{RCOH}\right]^{+} .{ }^{g}\left[\mathrm{M}+\mathrm{H}-2 \mathrm{H}_{2} \mathrm{O}-\right.$ $\mathrm{RCOH}]^{+} .{ }^{h}$ Other typical OL ion fragments. ${ }^{i}\left[\mathrm{M}+\mathrm{H}-2 \mathrm{H}_{2} \mathrm{O}\right] .{ }^{j}\left[\mathrm{M}+\mathrm{H}-3 \mathrm{H}_{2} \mathrm{O}-\mathrm{RCOH}\right]^{+} .{ }^{k}\left[\mathrm{M}+\mathrm{H}-\mathrm{C}_{2} \mathrm{H}_{8} \mathrm{NO}_{4} \mathrm{P}\right]^{+}$.

The molecular formulas of each VIP were proposed based on accurate mass measurement, true isotopic pattern, and fragmentation analysis. A detailed analysis of VIPs and molecular network revealed that most of these discriminating metabolites constitute the cluster A (Figure 4). These chemical compounds were specific to TC8, on one hand, and to TC4 and TC7, on the other hand. Interestingly, all of these specific compounds showed a similar fragmentation pattern with a characteristic ion fragment at $\mathrm{m} / z$ 115. A bibliographic review allowed us to propose ornithine-containing lipids (OL) as good candidates for this chemical group. OLs are widespread among Gram-negative bacteria, more rarely found in Gram-positive ones, and absent in eukaryotes and archaea. ${ }^{44}$ These membrane lipids contain an ornithine headgroup linked to a 3-hydroxy fatty acid via its $\alpha$-amino moiety and a second fatty acid chain (also called "piggyback" fatty acid) esterified to the hydroxyl group of the first fatty acid. In some bacteria the ester-linked fatty acid can be hydroxylated, usually at the C-2 position. ${ }^{45}$ OLs show a specific MS fragmentation pattern used in this study for their identification. Characteristic multistage MS fragmentation patterns include the sequential loss of $\mathrm{H}_{2} \mathrm{O}$ (from the ornithine part), the piggyback acyl chain, and the amide-linked fatty acid. ${ }^{46}$ This characteristic mode of fragmentation leads to headgroup fragment ions at $\mathrm{m} / z 159$ $\left(\mathrm{C}_{6} \mathrm{H}_{11} \mathrm{~N}_{2} \mathrm{O}_{3}\right), 141 \quad\left(\mathrm{C}_{6} \mathrm{H}_{9} \mathrm{~N}_{2} \mathrm{O}_{2}\right), 133 \quad\left(\mathrm{C}_{5} \mathrm{H}_{13} \mathrm{~N}_{2} \mathrm{O}_{2}\right), 115$
$\left(\mathrm{C}_{5} \mathrm{H}_{11} \mathrm{~N}_{2} \mathrm{O}_{2}\right)$, and $70\left(\mathrm{C}_{4} \mathrm{H}_{8} \mathrm{~N}\right)$. On that basis, HRMS/MS fragmentation of VIP no. $1(\mathrm{~m} / z 677)$ is proposed in Figure 5, and the same pattern was observed for the other OLs (Table 2). In Gram-negative bacteria, membranes are constituted by polar lipids frequently composed of phospholipids like phosphatidylethanolamine (PE). In this work, this type of lipids was detected in the four strains (cluster E, Figure 4), but several studies have shown that under phosphorus starvation, which is common in marine environments, the production of nonphosphorus polar lipids such as OLs may increase significantly. $^{47,48}$ Moreover, because of their zwitterionic character, OLs have been speculated to play a crucial role for the membrane stability of Gram-negative bacteria and more broadly for the adaptation of the membrane in response to changes of environmental conditions. Under the culture conditions used in this study, OLs were produced by three of the strains but not by Shewanella sp. TC11. In a same way, components of cluster B specifically produced by Bacteroidetes (TC4 and TC7) were identified as hydroxylated OLs (HOLs). These compounds showed the same MS fragmentation pattern as their nonhydroxylated analogs, while a supplementary loss of $\mathrm{H}_{2} \mathrm{O}$ was observed at the beginning of the MS fragmentation pathway. HOLs have been described as metabolites specifically produced by bacteria under stress (e.g., temperature, ${ }^{49} \mathrm{pH}^{50}$ ): the occurrence of an additional hydroxyl group seems to be 

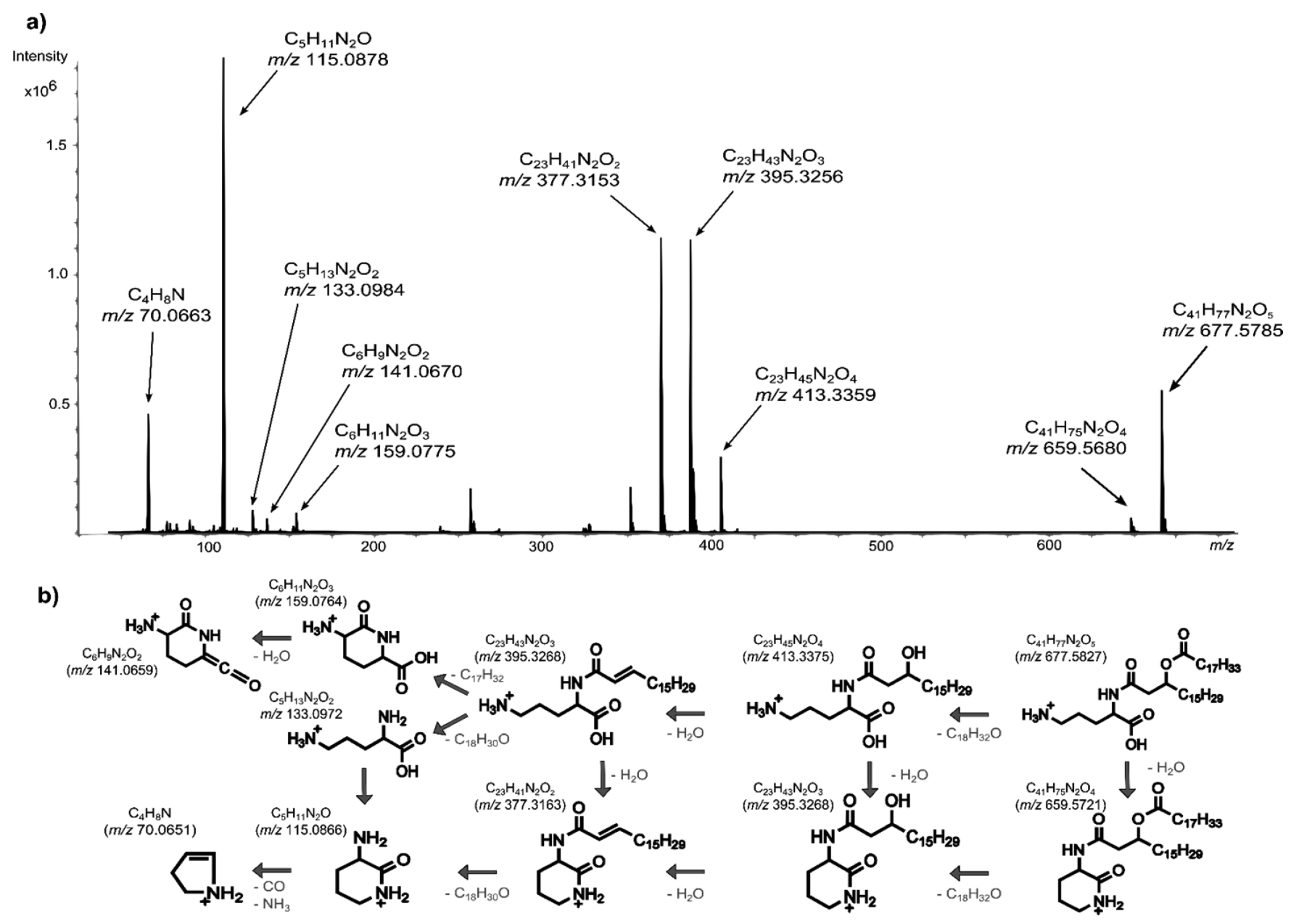

Figure 5. (a) HRMS mass spectra of Pseudoalteromonas lipolytica TC8 ornithine lipid at $m / z 677.5785$ (VIP no. 1). (b) Proposed fragmentation of VIP no. 1. (The elemental composition of fragment ions is indicated and the corresponding theoretical value of $m / z$ is given in parentheses.)

implied in the membranes stability via an increase in strong lateral interactions between their components. ${ }^{51}$ HOLs were mainly described in $\alpha$-, $\beta$-, and $\gamma$-proteobacteria and Bacteroidetes. $^{52}$ In our study HOLs were only detected in LC-MS profiles of Bacteroidetes (TC4 and TC7) but not in those of the $\gamma$-proteobacteria (TC8 and TC11). Concerning the position of the additional hydroxyl group in these derivatives, the absence of characteristic ion fragments for ornithine headgroup hydroxylation ${ }^{44}$ indicated that this group was linked to one of the two fatty acids (at the two-position). This structural feature was in agreement with the fact that hydroxylation of the ornithine headgroup in HOLs was only observed in $\alpha$-proteobacteria and not in Bacteroidetes. ${ }^{52}$ TC4 and TC7 were also clearly discriminated from the other strains through another class of metabolites putatively identified on the basis of their HRMS/MS data as glycine lipids (GLs) and close derivatives, namely, methylglycine or alanine lipids (cluster C, Figure 4). These compounds are structurally similar to OLs, the main difference being the replacement of the ornithine unit by a glycine one. They showed a similar fragmentation sequence and were specifically characterized by headgroup fragment ions at $m / z 76\left(\mathrm{C}_{2} \mathrm{H}_{6} \mathrm{NO}_{2}\right)$ for GLs and $m / z 90\left(\mathrm{C}_{3} \mathrm{H}_{8} \mathrm{NO}_{2}\right)$ for methylglycine or alanine lipids. This last class of compounds needs to be further confirmed by purification and full structure characterization. From a chemotaxonomic point of view, GLs are valuable compounds because they have only been described from Bacteroidetes and thus seem to be biomarkers of this bacterial group. ${ }^{52}$ Finally, when considering the discrimination between the two $P$. mediterranea strains, TC7 specifically produced a variety of lipids tentatively assigned as $\mathrm{N}$-acyl diamines by HRMS/MS (cluster D, Figure 4). More precisely, a fragmentation pattern common for most of the compounds of cluster $\mathrm{D}$ showed the occurrence of a diamine backbone with an amide-linked fatty acid and yielded fragment ions at $m / z 89$ $\left(\mathrm{C}_{4} \mathrm{H}_{13} \mathrm{~N}_{2}\right)$ and $72\left(\mathrm{C}_{4} \mathrm{H}_{10} \mathrm{~N}\right)$ characteristic of the putrescine headgroup. ${ }^{53}$ Several other chemical members of this cluster showed similar fragmentation pathways but with fragment ions, in accordance with slight variations of the chemical structure of the headgroup ( $N$-methylation, hydroxylation). In the case of TC7, compounds with a hydroxylated headgroup were specifically overexpressed. According to literature data, polyamines are commonly found in most living cells, and, among this chemical family, putrescine constitutes one of the simplest member. ${ }^{54}$ This diamine is widespread among bacteria and is involved in a large number of biological functions. ${ }^{55}$ Interestingly, ornithine can form putrescine either directly (ornithine decarboxylase) or indirectly from arginine (arginine decarboxylase) via agmatine (agmatine deiminase). Taking into account the few studies on MS fragmentation of natural $N$-acyl diamines and the absence of commercially available standards, further structure investigations are required to fully characterize this class of bacterial biomarkers and to establish a possible 
biosynthetic link with OLs. Finally, some other specific clusters were remarkable in the molecular network but without possible affiliation of the corresponding compounds to an existing chemical family. Conversely, molecular components of the nondiscriminative cluster $\mathrm{F}$ were putatively identified as cyclic dipeptides. This type of compounds exhibits a wide range of biological functions, and cyclic dipeptides are involved in chemical signaling in Gram-negative bacteria with a potential role in interkingdom communication. ${ }^{56-58}$

\section{CONCLUSIONS}

We described a metabolomics approach applied to the assessment of the effects of several culture parameters, such as culture media, growth phase, or mode of culture, in the metabolic discrimination between four marine biofilm-forming bacteria. The developed method based on a simple extraction protocol could differentiate bacterial strains cultured in organicrich media. Depending on the culture parameters, some significant intrastrain metabolic changes were observed, but overall these metabolome variations were always less pronounced than interstrain differences. Finally, several classes of biomarkers were putatively identified via HRMS/MS analysis and molecular networking. Under the culture conditions used (not phosphate-limited), OLs were thus identified as specifically produced by three of the bacteria, while HOLs and GLs were only detected in the two Bacteroidetes strains.

Our study provides evidence that such an analytical protocol is useful to explore more deeply the metabolome of marine bacteria under various culture conditions, including cultures in organic-rich media and biofilms. This efficient process gives information on the metabolome of marine bacterial strains that represent complementary data to those provided by genomic, transcriptomic, and proteomic analyses on the regulatory and metabolic pathways of marine bacteria involved in biofilms. Also, a broader coverage of the biofilm metabolome requires the examination of polar extracts even if high-salt contents limit drastically the analysis capabilities of polar compounds in marine bacterial cultures and environmental biofilm samples.

As an important result, bacterial acyl amino acids, and more broadly membrane lipids, can be used as efficient biomarkers not only for chemotaxonomy but also directly for studies directed toward bacterial stress response. Indeed, a targeted analysis of GLs would be efficient to estimate the occurrence of Bacteroidetes in complex natural biofilms, while OLs and HOLs would be valuable molecular tools to evaluate the response of bacteria to specific environmental conditions.

Moreover, to get closer to reality, future works in this specific field of research should address some more ecologically relevant questions. To this end, metabolomics studies involving multispecies cocultures or bacterial cultures implemented with signal compounds (e.g., $\mathrm{N}$-acyl-homoserine lactones, diketopiperazines) may be considered in the future and linked to similar data obtained from natural biofilms.

\section{ASSOCIATED CONTENT}

S Supporting Information

Figure S1. Growth stages of the four bacterial strains. Figure S2. Number of $m / z$ features detected for the four bacteria depending on the extraction solvent. Figure S3.
Venn diagrams showing unique and shared metabolites for the four bacterial strains in each extraction condition. Figure S4. PCA and PLS-DA score plots of the four bacterial strains in each extraction condition. Figure S5. Number of $m / z$ features detected for the four bacterial strains depending on the culture media. Figure S6. PCA score plots of the four bacterial strains cultured in two media and PLS-DA score plots of the four bacterial strains cultured in MB. Figure S7. Number of $\mathrm{m} / z$ features detected for each bacteria at five time points of the growth curve. Figure S8. PLS-DA score plots of TC8, TC4, and TC11 at five time points of their growth curve. Figure S9. PLS-DA score plots of TC7, TC8, and TC11 cultured in planktonic and biofilm modes. Figure S10. PCA and PLS-DA score plots of TC7, TC8, and TC11 cultured in biofilms. Figure S11. PCA score plots of TC7, TC8, and TC11 cultured in biofilms and planktonic conditions. Figure S12. PCA score plots (LC-HRMS) of the four bacterial strains. Figure S13. PLS-DA score plots (LC-HRMS) of TC8 at five time points of its growth curve. Table S1. Composition of the MB and VNSS culture media. Table S2. Parameters of the PLS-DA models used for the intrastrain discrimination depending on different culture conditions.(PDF)

\section{AUTHOR INFORMATION}

\section{Corresponding Author}

*Tel: (+33) 4941429 35. E-mail: culioli@univ-tln.fr. ORCID $\odot$

Olivier P. Thomas: 0000-0002-5708-1409

Gérald Culioli: 0000-0001-5760-6394

Notes

The authors declare no competing financial interest.

\section{ACKNOWLEDGMENTS}

This study was partly funded by the French "Provence-AlpesCôte d'Azur (PACA)” regional council (Ph.D. grant to L.F.). We are grateful to R. Gandolfo for the kind support of the French Mediterranean Marine Competitivity Centre (Pôle Mer Méditerranée) and thank J. C. Tabet, R. Lami, G. Genta-Jouve, J. F. Briand, and B. Misson for helpful discussions. LC-HRMS experiments were acquired on the regional platform MALLABAR (CNRS and PACA supports). Dedicated to Professor Louis Piovetti on the occasion of his 75th birthday.

\section{ABBREVIATIONS}

$\mathrm{ACN}$, acetonitrile; CS, cosine score; CV-ANOVA, cross validation-analysis of variance; DCM, dichloromethane; EtOAc, ethyl acetate; GC, gas-chromatography; GL, glycine lipids; GNPS, global natural product social molecular networking; HOL, hydroxylated ornithine lipid; HRMS, high resolution mass spectrometry; KEGG, Kyoto encyclopedia of genes and genomes; LC, liquid chromatography; LC-ESI-ITMS, liquid chromatography-electrospray ionization ion trap tandem mass spectrometry; LC-MS, liquid chromatographymass spectrometry; LRMS, low resolution mass spectrometry; $\mathrm{MB}$, marine broth; $\mathrm{MeOH}$, methanol; NMR, nuclear magnetic resonance; OL, ornithine lipid; PCA, principal component analysis; PE, phosphatidylethanolamine; PLS-DA, partial leastsquares discriminant analysis; TC, Toulon collection; UPLCESI-QToF-MS, ultraperformance liquid chromatography- 
electrospray ionization quadrupole time-of-flight tandem mass spectrometry; VIP, variable importance on projection; VNSS, Väätänen nine salt solution

\section{REFERENCES}

(1) Yebra, D. M.; Kiil, S.; Dam-Johansen, K. Antifouling technologyPast, present and future steps towards efficient and environmentally friendly antifouling coatings. Prog. Org. Coat. 2004, 50 (2), 75-104.

(2) Schultz, M. P.; Bendick, J. A.; Holm, E. R.; Hertel, W. M. Economic impact of biofouling on a naval surface ship. Biofouling 2011, 27 (1), 87-98.

(3) Railkin, A. I. Marine Biofouling: Colonization Processes and Defenses; CRC Press: Boca Raton, FL, 2004; p 376.

(4) Stoodley, P.; Sauer, K.; Davies, D. G.; Costerton, J. W. Biofilms as complex differentiated communities. Annu. Rev. Microbiol. 2002, 56 (1), 187-209.

(5) Flemming, H.-C.; Wingender, J.; Szewzyk, U.; Steinberg, P.; Rice, S. A.; Kjelleberg, S. Biofilms: an emergent form of bacterial life. Nat. Rev. Microbiol. 2016, 14 (9), 563-575.

(6) Matz, C.; Webb, J. S.; Schupp, P. J.; Phang, S. Y.; Penesyan, A.; Egan, S.; Steinberg, P.; Kjelleberg, S. Marine biofilm bacteria evade eukaryotic predation by targeted chemical defense. PLoS One 2008, 3 (7), e2744.

(7) Lau, S. C. K.; Thiyagarajan, V.; Cheung, S. C. K.; Qian, P.-Y. Roles of bacterial community composition in biofilms as a mediator for larval settlement of three marine invertebrates. Aquat. Microb. Ecol. 2005, 38 (1), 41-51.

(8) Dobretsov, S.; Qian, P.-Y. Facilitation and inhibition of larval attachment of the bryozoan Bugula neritina in association with monospecies and multi-species biofilms. J. Exp. Mar. Biol. Ecol. 2006, 333 (2), 263-274.

(9) Blunt, J. W.; Copp, B. R.; Keyzers, R. A.; Munro, M. H. G.; Prinsep, M. R. Marine natural products. Nat. Prod. Rep. 2016, 33 (3), 382-431.

(10) Dang, H.; Lovell, C. R. Microbial surface colonization and biofilm development in marine environments. Microbiol. Mol. Biol. Rev. 2016, 80 (1), 91-138.

(11) Zhou, B.; Xiao, J. F.; Tuli, L.; Ressom, H. W. LC-MS-based metabolomics. Mol. BioSyst. 2012, 8 (2), 470-481.

(12) Romano, S.; Dittmar, T.; Bondarev, V.; Weber, R. J. M.; Viant, M. R.; Schulz-Vogt, H. N. Exo-metabolome of Pseudovibrio sp. FOBEG1 analyzed by ultra-high resolution mass spectrometry and the effect of phosphate limitation. PLoS One 2014, 9 (5), e96038.

(13) Zech, H.; Thole, S.; Schreiber, K.; Kalhöfer, D.; Voget, S.; Brinkhoff, T.; Simon, M.; Schomburg, D.; Rabus, R. Growth phasedependent global protein and metabolite profiles of Phaeobacter gallaeciensis strain DSM 17395, a member of the marine Roseobacterclade. Proteomics 2009, 9 (14), 3677-3697.

(14) Takahashi, H.; Kai, K.; Shinbo, Y.; Tanaka, K.; Ohta, D.; Oshima, T.; Altaf-Ul-Amin, M.; Kurokawa, K.; Ogasawara, N.; Kanaya, S. Metabolomics approach for determining growth-specific metabolites based on Fourier transform ion cyclotron resonance mass spectrometry. Anal. Bioanal. Chem. 2008, 391 (8), 2769-2782.

(15) Brito-Echeverría, J.; Lucio, M.; López-López, A.; Antón, J.; Schmitt-Kopplin, P.; Rosselló-Móra, R. Response to adverse conditions in two strains of the extremely halophilic species Salinibacter ruber. Extremophiles 2011, 15 (3), 379-389.

(16) Brian-Jaisson, F.; Ortalo-Magné, A.; Guentas-Dombrowsky, L.; Armougom, F.; Blache, Y.; Molmeret, M. Identification of bacterial strains isolated from the Mediterranean sea exhibiting different abilities of biofilm formation. Microb. Ecol. 2014, 68 (1), 94-110.

(17) Kuehnbaum, N. L.; Britz-McKibbin, P. New advances in separation science for metabolomics: Resolving chemical diversity in a post-genomic era. Chem. Rev. 2013, 113 (4), 2437-2468.

(18) Gritti, F.; Guiochon, G. Performance of columns packed with the new shell Kinetex- $\mathrm{C}_{18}$ particles in gradient elution chromatography. J. Chromatogr. A 2010, 1217 (10), 1604-1615.
(19) Patti, G. J.; Tautenhahn, R.; Siuzdak, G. Meta-analysis of untargeted metabolomic data from multiple profiling experiments. Nat. Protoc. 2012, 7 (3), 508-516.

(20) Caraux, G.; Pinloche, S. PermutMatrix: a graphical environment to arrange gene expression profiles in optimal linear order. Bioinformatics 2005, 21 (7), 1280-1281.

(21) Kido Soule, M. C.; Longnecker, K.; Johnson, W. M.; Kujawinski, E. B. Environmental metabolomics: Analytical strategies. Mar. Chem. 2015, 177 (Part 2), 374-387.

(22) de Jonge, L. P.; Douma, R. D.; Heijnen, J. J.; van Gulik, W. M. Optimization of cold methanol quenching for quantitative metabolomics of Penicillium chrysogenum. Metabolomics 2012, 8 (4), 727735.

(23) Duportet, X.; Aggio, R. B. M.; Carneiro, S.; Villas-Bôas, S. G. The biological interpretation of metabolomic data can be misled by the extraction method used. Metabolomics 2012, 8 (3), 410-421.

(24) Shin, M. H.; Lee, D. Y.; Liu, K.-H.; Fiehn, O.; Kim, K. H. Evaluation of sampling and extraction methodologies for the global metabolic profiling of Saccharophagus degradans. Anal. Chem. 2010, 82 (15), 6660-6666.

(25) Canelas, A. B.; ten Pierick, A.; Ras, C.; Seifar, R. M.; van Dam, J. C.; van Gulik, W. M.; Heijnen, J. J. Quantitative evaluation of intracellular metabolite extraction techniques for yeast metabolomics. Anal. Chem. 2009, 81 (17), 7379-7389.

(26) Lu, L.; Wang, J.; Xu, Y.; Wang, K.; Hu, Y.; Tian, R.; Yang, B.; Lai, Q.; Li, Y.; Zhang, W.; Shao, Z.; Lam, H.; Qian, P.-Y. A highresolution LC-MS-based secondary metabolite fingerprint database of marine bacteria. Sci. Rep. 2014, 4, 6537.

(27) Bose, U.; Hewavitharana, A.; Ng, Y.; Shaw, P.; Fuerst, J.; Hodson, M. LC-MS-based metabolomics study of marine bacterial secondary metabolite and antibiotic production in Salinispora arenicola. Mar. Drugs 2015, 13 (1), 249.

(28) Vynne, N. G.; Månsson, M.; Nielsen, K. F.; Gram, L. Bioactivity, chemical profiling, and 16S rRNA-based phylogeny of Pseudoalteromonas strains collected on a global research cruise. Mar. Biotechnol. 2011, 13 (6), 1062-1073.

(29) Djinni, I.; Defant, A.; Kecha, M.; Mancini, I. Metabolite profile of marine-derived endophytic Streptomyces sundarbansensis WR1L1S8 by liquid chromatography-mass spectrometry and evaluation of culture conditions on antibacterial activity and mycelial growth. J. Appl. Microbiol. 2014, 116 (1), 39-50.

(30) Bode, H. B.; Bethe, B.; Höfs, R.; Zeeck, A. Big effects from small changes: Possible ways to explore Nature's chemical diversity. ChemBioChem 2002, 3 (7), 619-627.

(31) Drapal, M.; Perez-Fons, L.; Wheeler, P. R.; Fraser, P. D. The application of metabolite profiling to Mycobacterium spp.: Determination of metabolite changes associated with growth. J. Microbiol. Methods 2014, 106, 23-32.

(32) Jin, Y.-X.; Shi, L.-H.; Kawata, Y. Metabolomics-based component profiling of Halomonas sp. KM-1 during different growth phases in poly(3-hydroxybutyrate) production. Bioresour. Technol. 2013, 140, 73-79.

(33) Alam, M. T.; Merlo, M. E.; Hodgson, D. A.; Wellington, E. M.; Takano, E.; Breitling, R. Metabolic modeling and analysis of the metabolic switch in Streptomyces coelicolor. BMC Genomics 2010, 11 (1), 202.

(34) Herbst, F.-A.; Danielsen, H. N.; Wimmer, R.; Nielsen, P. H.; Dueholm, M. S. Label-free quantification reveals major proteomic changes in Pseudomonas putida F1 during the exponential growth phase. Proteomics 2015, 15 (18), 3244-3252.

(35) Sowell, S. M.; Norbeck, A. D.; Lipton, M. S.; Nicora, C. D.; Callister, S. J.; Smith, R. D.; Barofsky, D. F.; Giovannoni, S. J. Proteomic analysis of stationary phase in the marine bacterium "Candidatus Pelagibacter ubique. Appl. Environ. Microbiol. 2008, 74 (13), 4091-4100.

(36) Gade, D.; Gobom, J.; Rabus, R. Proteomic analysis of carbohydrate catabolism and regulation in the marine bacterium Rhodopirellula baltica. Proteomics 2005, 5 (14), 3672-3683. 
(37) Yeom, J.; Shin, J.-H.; Yang, J.-Y.; Kim, J.; Hwang, G.-S. ${ }^{1} \mathrm{H}$ NMR-based metabolite profiling of planktonic and biofilm cells in Acinetobacter baumannii 1656-2. PLoS One 2013, 8 (3), e57730.

(38) Ammons, M. C. B.; Tripet, B. P.; Carlson, R. P.; Kirker, K. R.; Gross, M. A.; Stanisich, J. J.; Copié, V. Quantitative NMR metabolite profiling of methicillin-resistant and methicillin-susceptible Staphylococcus aureus discriminates between biofilm and planktonic phenotypes. J. Proteome Res. 2014, 13 (6), 2973-2985.

(39) Chandramouli, K. H.; Dash, S.; Zhang, Y.; Ravasi, T.; Qian, P.-Y. Proteomic and metabolomic profiles of marine Vibrio sp. 010 in response to an antifoulant challenge. Biofouling 2013, 29 (7), 789802.

(40) Chavez-Dozal, A.; Gorman, C.; Nishiguchi, M. K. Proteomic and metabolomic profiles demonstrate variation among free-living and symbiotic vibrio fischeri biofilms. BMC Microbiol. 2015, 15 (1), 226.

(41) Hall-Stoodley, L.; Costerton, J. W.; Stoodley, P. Bacterial biofilms: from the Natural environment to infectious diseases. Nat. Rev. Microbiol. 2004, 2 (2), 95-108.

(42) Sauer, K. The genomics and proteomics of biofilm formation. Genome Biol. 2003, 4 (6), 219.

(43) Watrous, J.; Roach, P.; Alexandrov, T.; Heath, B. S.; Yang, J. Y.; Kersten, R. D.; van der Voort, M.; Pogliano, K.; Gross, H.; Raaijmakers, J. M.; Moore, B. S.; Laskin, J.; Bandeira, N.; Dorrestein, P. C. Mass spectral molecular networking of living microbial colonies. Proc. Natl. Acad. Sci. U. S. A. 2012, 109 (26), E1743-E1752.

(44) Moore, E. K.; Hopmans, E. C.; Rijpstra, W. I. C.; Villanueva, L.; Sinninghe Damsté, J. S. Elucidation and identification of amino acid containing membrane lipids using liquid chromatography/highresolution mass spectrometry. Rapid Commun. Mass Spectrom. 2016, 30 (6), 739-750.

(45) Geiger, O.; González-Silva, N.; López-Lara, I. M.; Sohlenkamp, C. Amino acid-containing membrane lipids in bacteria. Prog. Lipid Res. 2010, 49 (1), 46-60.

(46) Zhang, X.; Ferguson-Miller, S. M.; Reid, G. E. Characterization of ornithine and glutamine lipids extracted from cell membranes of Rhodobacter sphaeroides. J. Am. Soc. Mass Spectrom. 2009, 20 (2), 198212.

(47) Yao, M.; Elling, F. J.; Jones, C.; Nomosatryo, S.; Long, C. P.; Crowe, S. A.; Antoniewicz, M. R.; Hinrichs, K.-U.; Maresca, J. A. Heterotrophic bacteria from an extremely phosphate-poor lake have conditionally reduced phosphorus demand and utilize diverse sources of phosphorus. Environ. Microbiol. 2016, 18 (2), 656-667.

(48) Sandoval-Calderón, M.; Nguyen, D. D.; Kapono, C. A.; Herron, P.; Dorrestein, P. C.; Sohlenkamp, C. Plasticity of Streptomyces coelicolor membrane composition under different growth conditions and during development. Front. Microbiol. 2015, 6, e1465.

(49) Taylor, C. J.; Anderson, A. J.; Wilkinson, S. G. Phenotypic variation of lipid composition in Burkholderia cepacia: a response to increased growth temperature is a greater content of 2-hydroxy acids in phosphatidylethanolamine and ornithine amide lipid. Microbiology 1998, 144 (7), 1737-1745.

(50) Vences-Guzmán, M. Á.; Guan, Z.; Ormeño-Orrillo, E.; González-Silva, N.; López-Lara, I. M.; Martínez-Romero, E.; Geiger, O.; Sohlenkamp, C. Hydroxylated ornithine lipids increase stress tolerance in Rhizobium tropici CIAT899. Mol. Microbiol. 2011, 79 (6), $1496-1514$.

(51) Nikaido, H. Molecular basis of bacterial outer membrane permeability revisited. Microbiol. Mol. Biol. Rev. 2003, 67 (4), 593656.

(52) Sohlenkamp, C.; Geiger, O. Bacterial membrane lipids: Diversity in structures and pathways. FEMS Microbiol. Rev. 2016, 40 (1), 133159.

(53) Voynikov, Y.; Zheleva-Dimitrova, D.; Gevrenova, R.; Lozanov, V.; Zaharieva, M. M.; Tsvetkova, I.; Najdenski, H.; Yagi, S.; Almoulah, N. F.; Momekov, G. Hydroxycinnamic acid amide profile of Solanum schimperianum Hochst by UPLC-HRMS. Int. J. Mass Spectrom. 2016, $408,42-50$.
(54) Michael, A. J. Polyamines in eukaryotes, bacteria, and archaea. J. Biol. Chem. 2016, 291 (29), 14896-14903.

(55) Miller-Fleming, L.; Olin-Sandoval, V.; Campbell, K.; Ralser, M. Remaining mysteries of molecular biology: The role of polyamines in the cell. J. Mol. Biol. 2015, 427 (21), 3389-3406.

(56) Ryan, R. P.; Dow, J. M. Diffusible signals and interspecies communication in bacteria. Microbiology 2008, 154 (7), 1845-1858.

(57) Ortiz-Castro, R.; Díaz-Pérez, C.; Martínez-Trujillo, M.; del Río, R. E.; Campos-García, J.; López-Bucio, J. Transkingdom signaling based on bacterial cyclodipeptides with auxin activity in plants. Proc. Natl. Acad. Sci. U. S. A. 2011, 108 (17), 7253-7258.

(58) Holden, M. T. G.; Ram Chhabra, S.; De Nys, R.; Stead, P.; Bainton, N. J.; Hill, P. J.; Manefield, M.; Kumar, N.; Labatte, M.; England, D.; Rice, S.; Givskov, M.; Salmond, G. P. C.; Stewart, G. S. A. B.; Bycroft, B. W.; Kjelleberg, S.; Williams, P. Quorum-sensing cross talk: Isolation and chemical characterization of cyclic dipeptides from Pseudomonas aeruginosa and other Gram-negative bacteria. Mol. Microbiol. 1999, 33 (6), 1254-1266. 